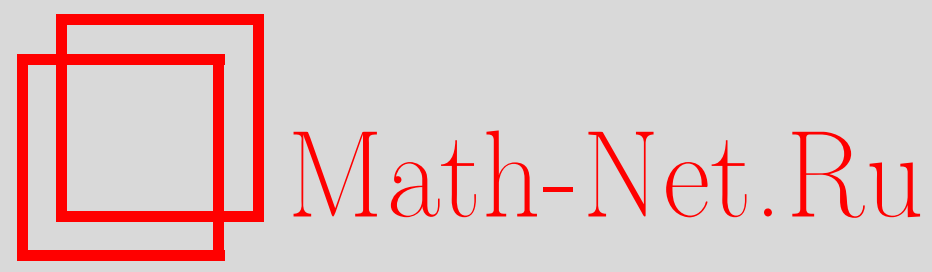

А. Б. Еловиков, Факторизация однопорожденных частично расслоенных формаций, Дискрет. матем., 2009, том 21, выпуск 3, 99-118

DOI: https://doi.org/10.4213/dm1064

Использование Общероссийского математического портала Math-Net.Ru подразумевает, что вы прочитали и согласны с пользовательским соглашением http://www.mathnet.ru/rus/agreement

Параметры загрузки:

IP: 3.85 .183 .62

26 апреля 2023 г., 05:27:21 


\title{
Факторизация однопорожденных частично расслоенных формаций
}

\author{
(ㄷ) 2009 г. А. Б. Еловиков
}

\begin{abstract}
Рассматриваются только конечные группы. Описан большой класс однопорожденных факторизуемых $\Omega$-расслоенных формаций, включая $\Omega$-композиционные, $\Omega$-биканонические и другие формации.
\end{abstract}

Одно из важных мест в теории формаций занимают вопросы, связанные с различными видами формационных произведений. Начало изучения факторизаций локальных формаций было положено в работах А. Н. Скибы и Л. А. Шеметкова $[1,2]$. В работе [3] А. Н. Скибой были описаны все нетривиальные факторизации однопорожденных локальных формаций. Разработанные в этих статьях методы и приемы исследования формаций конечных групп широко используются сейчас многими авторами в различных направлениях. В работах $[4,5]$ В. А. Ведерников предложил способ построения новых видов расслоенных формаций с помощью функции направления.

Концепция частичной локальности, введенная в рассмотрение Л. А. Шеметковым $[2,6]$ и концепция частичной расслоенности, введенная В. А. Ведерниковым $[5,7]$ позволили существенно расширить класс формаций, описываемых на языке функций. Поэтому естественным является интерес к изучению частично расслоенных формаций вообще и однопорожденных $\Omega$-расслоенных формаций, в частности. В статье [8] были изучены несократимые факторизации однопорожденных $\Omega$-расслоенных формаций целого ряда направлений с $\Omega=\mathfrak{F}$, частным случаем которых являются локальные, композиционные и биканонические формации. Данная работа завершает целостное исследование $\Omega$-расслоенных формаций описанием несократимых факторизаций частично расслоенных формаций ряда направлений с $\varnothing \subset \Omega \subset$ I, включающего в себя $\Omega$-композиционные и $\Omega$-биканонические формации.

Рассматриваются только конечные группы. Все необходимые определения и обозначения можно найти в $[9,10]$. Приведем только некоторые из них. Символами (ㄱ, , $\mathfrak{N}$, $\mathfrak{U}$ обозначают соответственно класс всех конечных групп, класс всех конечных разрешимых групп, класс всех конечных нильпотентных групп и класс всех конечных абелевых групп. Класс всех конечных простых групп обозначают $\mathfrak{\Im}$; в данной статье под $\Omega$ всегда будем понимать непустой подкласс класса $\mathfrak{s}$, который собственно содержится в $\mathfrak{s} ; \mathfrak{S}_{A}-$ класс всех конечных $A$-групп, то есть таких групп $G$, что $K(G)=(A)$, где $K(G)$ - класс всех простых групп, изоморфных композиционным факторам группы $G ; f: \Omega \cup\left\{\Omega^{\prime}\right\} \rightarrow\{$ формации групп\}, $\varphi: \mathfrak{I} \rightarrow\{$ непустые формации Фиттинга $\}-\Omega F$ функция и $F R$-функция соответственно. Все рассматриваемые функции принимают одинаковые значения на изоморфных группах из их области определения. Формация

$$
\mathfrak{F}=\Omega F(f, \varphi)=\left(G: G / O_{\Omega}(G) \in f\left(\Omega^{\prime}\right)\right.
$$


и $G / G_{\varphi(A)} \in f(A)$ для всех $\left.A \in \Omega \cap K(G)\right)$ называется $\Omega$-расслоенной формацией с $\Omega$-спутником $f$ и с направлением $\varphi$ (см. [5]). Зафиксируем $\alpha(\gamma)-$ направление биканонической (композиционной) формации, то есть $\alpha(A)=\mathbb{G}_{A^{\prime}}$ для любой неабелевой группы $A \in \mathfrak{s}$ и $\alpha(A)=\mathbb{S}_{A^{\prime}} \mathfrak{\Im}_{A}$ для любой абелевой группы $A \in \mathfrak{s}\left(\gamma(A)=\mathbb{S}_{c A}\right.$ для всех групп $A \in \mathfrak{I})$. Направление $\varphi \Omega$-расслоенной формации называют $b$-направлением, если $\varphi(A) \mathcal{G}_{A}=\varphi(A)$ для любой абелевой группы $A \in \mathfrak{s} ; r$-направлением, если $\varphi(A)=\mathfrak{\Im}_{A^{\prime}} \varphi(A)$ для любого $A \in \mathfrak{s} ; b r$-направлением, если $\varphi$ является и $b$-направлением и $r$-направлением (см. [5]). На множестве $\Re$ всех $F R$-функций вводится отношение частичного порядка $\leqslant$. Для любых $\varphi_{1}, \varphi_{2} \in \Re$ полагают $\varphi_{1} \leqslant \varphi_{2}$, если $\varphi_{1}(A) \subseteq \varphi_{2}(A)$ для любого $A \in \mathfrak{S}$. В данной статье под направлением $\beta$ всегда понимается некоторое $b r$-направление такое, что $\alpha \leqslant \beta \leqslant \gamma$.

Через $G=[A] B$ обозначают полупрямое произведение групп $A$ и $B$, где $A$ - нормальная подгруппа в $G$. Остальные нестандартные обозначения будут вводиться по ходу изложения материала.

Используя методы работы [5], несложно показать, что справедливо следующее утверждение.

Лемма 1. Пусть $f_{1} u f_{2}$ - внутренние $\Omega$-спутники $\Omega$-расслоенной формаџии $\mathfrak{F}$ с направлением $\beta$. Тогда

(1) $\mathfrak{\Im}_{A} f_{1}(A) \subseteq \mathfrak{F}$ для любой $A \in \Omega \cap \mathfrak{U}$;

(2) $\varsigma_{A} f_{1}(A)=\varsigma_{A} f_{2}(A)$ для любой $A \in \Omega \cap \mathfrak{U}$;

(3) $\mathfrak{F}$ обладает внутренним $\Omega$-спутником $g$ таким, что $g(A)=\mathfrak{F}$, если А принадлежит $(\Omega \backslash \mathfrak{U}) \cup\left\{\Omega^{\prime}\right\}$ и $g(A)=\varsigma_{A} f_{1}(A)$ для любой $A \in \Omega \cap \mathfrak{U}$.

Из леммы 1 непосредственно вытекает следующее утверждение.

Лемма 2. Пусть $f$-внутренний $\Omega$-спутник $\Omega$-расслоенной формациии $\mathfrak{F}$ с направлением $\beta$. Тогда формация $\mathfrak{F}$ имеет единственный максимальный внутренний $\Omega$-спутник $h$, причем $h(A)=\mathfrak{F}$, если $A \in(\Omega \backslash \mathfrak{U}) \cup\left\{\Omega^{\prime}\right\}$ и $h(A)=\mathfrak{F}_{A} h(A)=\mathfrak{G}_{A} f(A)$ для любой $A \in \Omega \cap \mathfrak{A}$.

Лемма 3. Пусть $\mathfrak{F}$ - наследственная $\Omega$-расслоенная формация с направлением $\beta$, тогда $f(A)$ также наследственна для всех $A \in \Omega \cup\left\{\Omega^{\prime}\right\}$, где $f-$ максимальный внутренний $\Omega$-спутник формации $\mathfrak{F}$.

Доказательство. По лемме $2 f(A)=\mathfrak{F}-$ наследственная формация для любой $A \in(\Omega \backslash \mathfrak{U}) \cup\left\{\Omega^{\prime}\right\}$.

Допустим, что существует $A \in \Omega \cap \mathfrak{U}$ такая, что найдется $G \in f(A), M<G$ и $M \notin f(A)$, причем $G$ - группа минимального порядка с таким свойством. Тогда $G \neq 1$. Если группа $G$ не монолитическая, то в $G$ существуют две различные минимальные нормальные подгруппы $R$ и $N$, причем $G / R \in f(A)$ и $G / N \in f(A)$. По индукции $M R / R \in f(A)$ и $M N / N \in f(A)$, а значит, $M /(M \cap R \cap N) \cong M \in f(A)$. Получаем противоречие. Следовательно, $G$ - монолитическая группа с монолитом $R$. Допустим, что $O_{A}(G) \neq 1$. Тогда $R \subseteq O_{A}(G)=O_{p}(G)$ для некоторого простого числа $p$ и $M R / R \in f(A)$. Но в этом случае, по лемме $2 M \in \varsigma_{A} f(A)=f(A)$. Получаем противоречие. Значит, $O_{A}(G)=1$.

По лемме 18.8 из [9] существует точный неприводимый $F_{p}[G]$-модуль $T$. Рассмотрим $\Gamma=[T] G$. Поскольку $\Gamma / T \cong G \in f(A) \subseteq \mathfrak{F}$ и $O_{p}(\Gamma) \subseteq \Gamma_{\beta(A)}$, по лемме 2 из [5] 
$\Gamma \in \mathfrak{F}$. Так как $T M<\Gamma$, по условию $T M \in \mathfrak{F}$. Тогда $T M /(T M)_{\beta(A)} \in f(A)$. Поскольку $C_{\Gamma}(T)=T=C_{T M}(T)-p$-группа, справедливы равенства

$$
(T M)_{\gamma(A)}=F_{A}(T M)=O_{p}(T M) .
$$

Значит,

$$
(T M)_{\beta(A)}=O_{p}(T M)=O_{p}(T M) \cap T M=T\left(O_{p}(T M) \cap M\right) \subseteq T O_{p}(M) .
$$

Таким образом,

$$
T M /\left(T O_{p}(M)\right) \cong M /\left(M \cap T O_{p}(M)\right) \cong M /\left(O_{p}(M)(M \cap T)\right)=M / O_{p}(M) \in f(A)
$$

и $M \in \mathfrak{G}_{A} f(A)=f(A)$. Получаем противоречие. В силу произвольности выбора $A$, $f(A)$ - наследственная формация для всех $A \in \Omega \cap \mathfrak{U}$. Лемма доказана.

Непустое множество формаций $\theta$ называется полной решеткой формаций, если $\varnothing$, \& $\in \theta$ и пересечение любой совокупности формаций из $\theta$ снова принадлежит $\theta$ (см. [6]). Через $\Omega \theta F(\mathfrak{X}, \beta)$ обозначим пересечение всех $\Omega$-расслоенных формаций с направлением $\beta$, содержащих класс групп $\mathfrak{X}$ и обладающих хотя бы одним $\theta$-значным $\Omega$-спутником.

Лемма 4. Пусть $\mathfrak{X}$ - непустой класс групп и $\theta-$ полная решетка формаций. Тогда формация $\mathfrak{F}=\Omega \theta F(\mathfrak{X}, \beta)$ обладает единственным минимальнылм $\theta$-значным $\Omega$-спутником $f$, причем

$$
f\left(\Omega^{\prime}\right)=\theta \operatorname{form}\left(G / O_{\Omega}(G): G \in \mathfrak{X}\right), \quad f(A)=\theta \text { form }\left(G / G_{\beta(A)}: G \in \mathfrak{X}\right)
$$

для всех $A \in K(\mathfrak{X}) \cap \Omega$ и $f(A)=\varnothing$, если $A \in \Omega \backslash K(\mathfrak{X})$.

Доказательство проводится аналогично доказательству теоремы 5 в [4].

Следствие 1. Пусть $f_{i}$ - минимальный $\theta$-значный $\Omega$-спутник $\Omega$-расслоенной формации $\mathfrak{F}_{i}$ с направлением $\beta, i=1,2$. Тогда и только тогда $\mathfrak{F}_{1} \subseteq \mathfrak{F}_{2}$, когда $f_{1} \leqslant f_{2}$.

Следствие 2. Пусть $f_{i}-$ максимальный внутренний $\theta$-значный $\Omega$-спутник $\Omega$-расслоенной формации $\mathfrak{F}_{i}$ с направлением $\beta, i=1,2$. Тогда и только тогда $\mathfrak{F}_{1} \subseteq \mathfrak{F}_{2}$, когда $f_{1} \leqslant f_{2}$.

Следствие 3. Пусть $\mathfrak{F}=\Omega F(\mathfrak{X}, \beta)$, где $\mathfrak{X}-$ непустой класс групп. Тогда

$$
K(\mathfrak{F}) \cap \Omega=K(\mathfrak{X}) \cap \Omega .
$$

Лемма 5. В однопорожденной $\Omega$-расслоенной формациии $\Omega F(G, \beta)$ содержится лишь конечное множество наследственных $\Omega$-расслоенных подформаций с направлением $\beta$.

Доказательство. Обозначим $\theta$ полную решетку всех наследственных формаций. Пусть $\mathfrak{M}=\Omega \theta F(G, \beta)$ - пересечение всех $\Omega$-расслоенных формаций с направлением $\beta$, содержащих $G$ и имеющих хотя бы один $\theta$-значный $\Omega$-спутник. Очевидно, что $\Omega F(G, \beta) \subseteq \mathfrak{M}$.

Пусть $\mathfrak{F}$ - произвольная наследственная $\Omega$-расслоенная формация с направлением $\beta$ такая, что $\mathfrak{F} \subseteq \mathfrak{M}$. В силу леммы 3 формация $\mathfrak{F}$ имеет хотя бы один $\theta$-значный $\Omega$-спутник. Пусть $f$ и $m$ - минимальные наследственные $\Omega$-спутники формаций $\mathfrak{F}$ и $\mathfrak{M}$ соответственно, тогда по следствию 1 справедливо неравенство $f \leqslant m$. По лемме 4 справедливо равенство $m(A)=\varnothing$, если $A \in \Omega \backslash K(G), m(A)=\theta \operatorname{form}\left(G / G_{\beta(A)}\right)$ для всех $A \in K(G) \cap \Omega$ и $m\left(\Omega^{\prime}\right)=\theta \operatorname{form}\left(G / O_{\Omega}(G)\right)$ - однопорожденные формации. Следовательно, по лемме 8.8 из [9] существует лишь конечное множество $\theta$-значных $\Omega$-спутников $t<m$. Значит в $\mathfrak{M}$, а тем более в $\Omega F(G, \beta)$, существует лишь конечное множество наследственных $\Omega$-расслоенных формаций с направлением $\beta$. Лемма доказана. 
Лемма 6. Если $M-$ произвольная подгруппа группь $G \in \Re_{\omega} \Re$, то $M \in \Omega F(G, \beta)$, где $\omega=\pi(\Omega \cap \mathfrak{U})$.

Доказательство. Если $G \in \Re$, то в силу теоремы 2.4 из [10] справедливо включение $M \in \Omega F(G, \beta)$. Предположим, что $G \notin \mathfrak{\Re}$. Тогда $G^{\mathfrak{N}} \in \mathfrak{N}_{\omega}$ и $G^{\mathfrak{N}} \neq 1$.

Проведем индукцию по порядку группы $G$. Пусть существует подгруппа $M$ группы $G$ такая, что $M \notin \mathfrak{F}=\Omega F(G, \beta)$. Причем $G$ - группа наименьшего порядка с таким свойством. Ясно, что $G-$ монолитическая группа с монолитом $R$. Поскольку $G^{\mathfrak{N}} \in \mathfrak{N}_{\omega}$, монолит $R$ - элементарная абелева $p$-группа для некоторого $p \in \omega$.

По индукции $M /(M \cap R) \in \mathfrak{F}$. Но $M \cap R \subseteq O_{p}(M)$, поэтому $M / O_{p}(M) \in \mathfrak{F}$. Учитывая теорему 4 из [4], несложно показать, что $\mathfrak{F}-\Omega$-композиционная формация. Таким образом,

$$
G_{\beta\left(Z_{p}\right)}=F_{Z_{p}}(G)=O_{\left(Z_{p}\right)^{\prime}, Z_{p}}(G)=O_{p}(G) .
$$

Обозначим через $f$ максимальный внутренний $\Omega$-спутник формации $\Omega F(G, \beta)$. По индукции

$$
M O_{p}(G) / O_{p}(G) \cong M /\left(M \cap O_{p}(G)\right) \in \Omega F\left(G / O_{p}(G), \beta\right) \subseteq f\left(Z_{p}\right)
$$

Тогда, по лемме $2, M \in \Re_{p} f\left(Z_{p}\right)=f\left(Z_{p}\right)$ и $M / M_{\beta\left(Z_{p}\right)} \in f\left(Z_{p}\right)$. В силу леммы 2 из [5] справедливо включение $M \in \mathfrak{F}$. Получаем противоречие. Лемма доказана.

Лемма 7. Если $\mathfrak{F}=\mathfrak{M} \mathfrak{5}$, где $\mathfrak{F}$, $\mathfrak{M}$ - неединичные $\Omega$-расслоенные формации с направлением $\beta$, а $\mathfrak{H}$ - такая формачия, что $K(\mathfrak{H}) \cap \Omega \cap \mathfrak{U} \nsubseteq K(\mathfrak{M}) \cap \Omega \cap \mathfrak{U} \neq \varnothing$, то множество наследственных $\Omega$-расслоенных подформаџий с направлением $\beta$ формации $\mathfrak{F}$ бесконечно.

Доказательство. Пусть $\left.Z_{p} \in K(\mathfrak{S}) \cap \Omega\right) \backslash K(\mathfrak{M})$. Ясно, что $\mathfrak{H} \subseteq \mathfrak{F}$. Значит, справедливо включение $Z_{p} \in K(\mathfrak{F}) \cap \Omega$. Из леммы 1 следует, что $\mathfrak{N}_{p} \subseteq \mathfrak{F}$. Пусть $A-$ произвольная группа из $\mathfrak{N}_{p}$. Так как $A \in \mathfrak{F}=\mathfrak{M} \mathfrak{H}$, справедливо включение $A^{\mathfrak{S}} \in \mathfrak{M}$. Но $Z_{p} \notin K(\mathfrak{M})$. Поэтому $A \in \mathfrak{H}$. Таким образом, $\mathfrak{N}_{p} \subseteq \mathfrak{H}$. Пусть $Z_{q} \in K(\mathfrak{M}) \cap \Omega$. Тогда $q \neq p$ и $\mathfrak{N}_{q} \subseteq \mathfrak{M}$, поскольку $\mathfrak{M}-\Omega$-расслоенная формация. Для любого натурального числа $n$ зафиксируем некоторую циклическую группу $P_{n}$ порядка $p^{n}$. Построим регулярное сплетение $Q_{n}=Z_{q}$ 2 $P_{n}$. Поскольку $Q_{n} \in \mathfrak{N}_{q} \mathfrak{N}_{p} \subseteq \mathfrak{M} \mathfrak{S}=\mathfrak{F}$, справедливо соотношение $\mathfrak{F}_{n}=\Omega F\left(Q_{n}, \beta\right) \subseteq \mathfrak{F}$. Кроме того, $\mathfrak{F}_{n} \subseteq \mathfrak{N}_{\omega} \mathfrak{N}$. Значит, в силу леммы $6, \mathfrak{F}_{n}-$ наследственная $\Omega$-расслоенная подформация в $\mathfrak{F}$.

При доказательстве леммы 8.13 в [9] было показано, что если $n$ и $m-$ различные натуральные числа, то $\mathfrak{F}_{n} \neq \mathfrak{F}_{m}$. Лемма доказана.

Лемма 8. Пусть $\mathfrak{M}=\Omega F(m, \beta)$, где $m-$ некоторый внутренний $\Omega$-спутник формации $\mathfrak{M}$, и $\mathfrak{H}$ - непустая формачия, причем $K(\mathfrak{S}) \cap \mathfrak{U} \cap \Omega \subseteq K(\mathfrak{M})$. Тогда $\mathfrak{F}=\mathfrak{M} \mathfrak{H}=\Omega F(f, \beta)$, где $f(A)=m(A) \mathfrak{H}$, если $A \in K(\mathfrak{M}) \cap \Omega ; f(A)=\mathfrak{H}$, если $A \in(\Omega \cap K(\mathfrak{H})) \backslash K(\mathfrak{M})$; $f(A)=\varnothing$ для всех $A \in \Omega \backslash(K(\mathfrak{S}) \cup K(\mathfrak{M}))$ и $f\left(\Omega^{\prime}\right)=m\left(\Omega^{\prime}\right) \mathfrak{H}$.

Доказательство. Пусть $\mathfrak{F}_{1}=\Omega F(f, \beta)$. Возьмем произвольную группу $G$ из $\mathfrak{F}$. Поскольку $G \in \mathfrak{F}$, справедливо равенство $G / O_{\Omega}(G) \in \mathfrak{F}=\mathfrak{M} \mathfrak{j}$. Тогда

$$
\left(G / O_{\Omega}(G)\right)^{\mathfrak{H}} \cong G^{\mathfrak{H}} /\left(G^{\mathfrak{H}} \cap O_{\Omega}(G)\right)=G^{\mathfrak{H}} / O_{\Omega}\left(G^{\mathfrak{H}}\right) \in \mathfrak{M} .
$$

Но в этом случае $O_{\Omega}\left(G^{\mathfrak{H}} / O_{\Omega}\left(G^{\mathfrak{H}}\right)\right)=1$ и $G^{\mathfrak{H}} / O_{\Omega}\left(G^{\mathfrak{H}}\right) \in m\left(\Omega^{\prime}\right)$. Поэтому

$$
G / O_{\Omega}(G) \in m\left(\Omega^{\prime}\right) \mathfrak{H}=f\left(\Omega^{\prime}\right) .
$$


Если $K(G) \cap \Omega=\varnothing$, то $G / O_{\Omega^{\prime}}(G) \cong 1 \in \mathfrak{F}_{1}$ и $G \in \mathfrak{F}_{1}$ в силу леммы 2 из [5]. Допустим, что $K(G) \cap \Omega \neq \varnothing$. В случае, когда $A \in K\left(G^{\mathfrak{5}}\right) \cap \Omega, A$ принадлежит $K(\mathfrak{M}) \cap \Omega$. Из свойств радикала и корадикала получаем, что

$$
\left(G / G_{\beta(A)}\right)^{\mathfrak{H}} \cong G^{\mathfrak{H}} /\left(G_{\beta(A)} \cap G^{\mathfrak{H}}\right)=G^{\mathfrak{H}} /\left(G^{\mathfrak{H}}\right)_{\beta(A)} \in m(A),
$$

поэтому $G / G_{\beta(A)} \in m(A) \mathfrak{H}=f(A)$. Пусть $A \in(K(G) \cap \Omega) \backslash K\left(G^{\mathfrak{H}}\right)$. Таким образом, $G^{\mathfrak{H}} \subseteq O_{A^{\prime}}(G) \subseteq G_{\alpha(A)} \subseteq G_{\beta(A)}$. Тогда из того, что $G / G^{\mathfrak{S}} \in \mathfrak{H}$, следует включение $G / G_{\beta(A)} \in \mathfrak{H} \subseteq f(A)$. Итак, $G / G_{\beta(A)} \in f(A)$ для любой $A \in K(G) \cap \Omega$. Следовательно, $G \in \mathfrak{F}_{1}$. Значит $\mathfrak{F} \subseteq \mathfrak{F}_{1}$.

Пусть $G \in \mathfrak{F}_{1} \backslash \mathfrak{F}$ и $G$ - группа минимального порядка с таким свойством. Тогда группа $G$ монолитическая с монолитом $R=G^{\mathfrak{r}}$.

Из того, что $\mathfrak{H} \subseteq \mathfrak{F}$ и $G \notin \mathfrak{F}$, следует, что $G \notin \mathfrak{H}$. Значит, $G^{\mathfrak{S}} \neq 1$ и из монолитичности $G$ следует, что $R \subseteq G^{\mathfrak{S}}$. Поскольку $G / R \in \mathfrak{F}=\mathfrak{M} 5$, справедливо включение $(G / R)^{\mathfrak{H}} \in \mathfrak{M}$, и следовательно,

$$
(G / R)^{\mathfrak{H}} \cong G^{\mathfrak{H}} /\left(R \cap G^{\mathfrak{H}}\right)=G^{\mathfrak{H}} / R \in \mathfrak{M} .
$$

Допустим, что $R$ не является $\Omega$-группой, тогда $O_{\Omega}(G)=1$, а значит,

$$
G \cong G / O_{\Omega}(G) \in f\left(\Omega^{\prime}\right) \subseteq \mathfrak{F} .
$$

Получаем противоречие. Следовательно, $R$ является $\Omega$-группой и $K(R)=(A)$. Если $A \in K(\mathfrak{M})$, то $G / G_{\beta(A)} \in m(A) \mathfrak{H}$, то есть

$$
\left(G / G_{\beta(A)}\right)^{\mathfrak{H}} \cong G^{\mathfrak{H}} /\left(G^{\mathfrak{H}}\right)_{\beta(A)} \in m(A) .
$$

Так как $G^{\mathfrak{H}} / R \in \mathfrak{M}$, из леммы 2 , доказанной в [5], следует, что $G^{\mathfrak{H}} \in \mathfrak{M}$, а значит, $G \in \mathfrak{M S}=\mathfrak{F}$. Получаем противоречие. Пусть $A \in \Omega \backslash K(\mathfrak{M})$. Тогда справедливы включения $G / G_{\beta(A)} \in f(A) \neq \varnothing$ и $A \in(\Omega \cap K(\mathfrak{H})) \backslash K(\mathfrak{M})$. Из условия теоремы следует, что $A$ - простая неабелева группа. Поэтому

$$
G / G_{\beta(A)}=G / O_{A^{\prime}}(G) \cong G \in f(A)=\mathfrak{H} \subseteq \mathfrak{F} .
$$

Получаем противоречие. Значит, $\mathfrak{F}_{1} \subseteq \mathfrak{F}$. Таким образом, $\mathfrak{F}=\mathfrak{F}_{1}$. Лемма доказана.

Будем обозначать через $\triangle(\mathfrak{F})$ множество всех попарно неизоморфных групп из $K(\mathfrak{F})$.

Лемма 9. Тогда и только тогда $\Omega$-расслоенная непустая формация $\mathfrak{F}$ с направлением $\beta$ является однопорожденной $\Omega$-расслоенной формацией, когда $\triangle(\mathfrak{F}) \cap \Omega-$ конечное множество и $\mathfrak{F}$ обладает таким внутренним $\Omega$-спутником $f$, что непустая формация $f(A)$ однопорожденна для всех $A \in \Omega \cup\left\{\Omega^{\prime}\right\}$.

Доказательство. Необходимость следует из теоремы 5 в [4]. Докажем достаточность. Пусть

$$
\triangle(\mathfrak{F}) \cap \Omega=\left\{A_{1}, A_{2}, \ldots, A_{t}\right\}
$$

и существует внутренний $\Omega$-спутник $f$ такой, что $f(A)$ - однопорожденная формация для всех $A \in \Omega \cup\left\{\Omega^{\prime}\right\}$. В частности, $f\left(A_{i}\right)=\operatorname{form}\left(G_{i}\right), i \in\{1,2, \ldots, t\}$, и $f\left(\Omega^{\prime}\right)=\operatorname{form}\left(G_{0}\right)$. Без ограничения общности, можем считать, что $A_{1}, A_{2}, \ldots, A_{n}$ - абелевы группы, $n \leqslant t$, 
то есть $A_{i} \cong Z_{p_{i}}$ для некоторого простого $p_{i}$, а $A_{n+1}, \ldots, A_{t}-$ простые неабелевы группы. Для всякого $i \in\{1,2, \ldots n\}$ рассмотрим регулярное сплетение

$$
B_{i}=A_{i} \prec\left(G_{i} / O_{A_{i}}\left(G_{i}\right)\right)=\left[K_{i}\right]\left(G_{i} / O_{A_{i}}\left(G_{i}\right)\right),
$$

где $K_{i}-$ база сплетения.

Пусть

$$
G=G_{0} \times B_{1} \times \ldots \times B_{n} \times G_{n+1} \times \ldots \times G_{t}
$$

и $\mathfrak{M}=\Omega F(G, \beta)$. Обозначим через $m$ максимальный внутренний $\Omega$-спутник формации $\mathfrak{M}$. Поскольку $f$ - внутренний $\Omega$-спутник формации $\mathfrak{F}$, справедливы включения $G_{i} \in \mathfrak{F}$, $i \in\{0,1,2, \ldots t\}$. Более того, $B_{i} / K_{i} \cong G_{i} / O_{A_{i}}\left(G_{i}\right) \in f\left(A_{i}\right)$ и в силу леммы 1 справедливы включения $B_{i} \in \mathbb{S}_{A_{i}} f\left(A_{i}\right) \subseteq \mathfrak{F}$ для всех $i \in\{1,2, \ldots n\}$. Итак, $\mathfrak{M} \subseteq \mathfrak{F}$.

Из леммы 2 следует, что $f\left(A_{i}\right) \subseteq m\left(A_{i}\right)=\mathfrak{M}$ для всех $i \in\{n+1, \ldots, t\}$ и $f\left(\Omega^{\prime}\right) \subseteq m\left(\Omega^{\prime}\right)=\mathfrak{M}$. Кроме того $B_{i} /\left(B_{i}\right)_{\beta\left(A_{i}\right)} \in m\left(A_{i}\right)$ для $i \in\{1,2, \ldots, n\}$. Заметим, что $\left(B_{i}\right)_{\beta\left(A_{i}\right)}=K_{i}$ для $i \in\{1,2, \ldots, n\}$. Поэтому $B_{i} / K_{i} \cong G_{i} / O_{A_{i}}\left(G_{i}\right) \in m\left(A_{i}\right)$ для всех $i \in\{1,2, \ldots, n\}$. Таким образом, $G_{i} \in \mathcal{G}_{A_{i}} m\left(A_{i}\right)=m\left(A_{i}\right)$ для $i \in\{1,2, \ldots, n\}$. В итоге получаем, что $f \leqslant m$ и $\mathfrak{F} \subseteq \mathfrak{M}$. Лемма доказана.

Следующее утверждение доказывается аналогично лемме 13 в [8].

Лемма 10. Пусть $h-$ произвольный $\Omega$-спутник $\Omega$-расслоенной формаџии $\mathfrak{F}$ с направлением $\beta$. Тогда для минимального внутреннего $\Omega$-спутника $f$

$$
f(A)=\operatorname{form}(G: G \in h(A) \cap \mathfrak{F}
$$

и $O_{B}(G)=1$ для всех $\left.B \in(K(\mathfrak{F}) \cap \Omega) \backslash(A)\right)$, если $A-$ неабелева группа из $\Omega \cap K(\mathfrak{F}), u$

$$
f(A)=\operatorname{form}\left(G: G \in h(A) \cap \mathfrak{F}, O_{A}(G)=1\right)
$$

для абелевой группь $A \in \Omega \cap K(\mathfrak{F})$.

Лемма 11. Пусть $\mathfrak{F}=\mathfrak{M S}-$ однопорожденная $\Omega$-расслоенная формация с направлением $\beta$, причем $\mathfrak{M}=\Omega F(m, \beta)$, где $m-$ внутренний $\Omega$-спутник формации $\mathfrak{M}$, а $\mathfrak{H}-$ неединичная формация. Тогда, если найдется такая группа $Z_{p} \in \Omega \cap \mathfrak{U}$, что в $m\left(Z_{p}\right)$ имеется простая группа А такая, что $|A| \neq p$, то $A \notin \mathfrak{H}$. Более того, если $A-$ простая абелева группа, то формащия $\mathfrak{5}$ абелева.

Доказательство. По условию $\mathfrak{F}=\Omega F(G, \beta)$ для некоторой группы $G$. Обозначим через $f$ минимальный $\Omega$-спутник формации $\mathfrak{F}$.

Предположим, что $A \in \mathfrak{S} \backslash \mathfrak{A}$. Рассмотрим группу $W=A \prec A^{m}$, где $A^{m}$ - прямое произведение $m$ экземпляров группы $A, m=|G|$. По лемме 8 , формация $\mathfrak{F}$ имеет такой внутренний $\Omega$-спутник $t$, что $t\left(Z_{p}\right)=m\left(Z_{p}\right) \mathfrak{H}$. Несложно показать, что $W \in m\left(Z_{p}\right) \mathfrak{H}=t\left(Z_{p}\right)$. Из леммы 10 вытекает, что $W \in f\left(Z_{p}\right)=\operatorname{form}\left(G / G_{\beta\left(Z_{p}\right)}\right)$. По лемме 3.1 .9 из [12], группа $W$ монолитическая с монолитом порядка $|A|^{|A|^{m}}$. Получено противоречие с леммой 3.1 .5 из [12]. Следовательно, $A$ - простая абелева группа. Согласно лемме 3.1.7 из [12], ступень сплетения $W$ не меньше $m+1$, что противоречит лемме 3.1 .5 из [12]. Таким образом, $A \notin \mathfrak{H}$.

Пусть $A-$ простая абелева группа порядка $q \neq p$. Допустим, что $q \in \pi(\mathfrak{H})$. Пусть $H$ - некоторая $q d$-группа из $\mathfrak{S}$. Для всякого натурального числа $n$ через $H_{n}$ обозначим регулярное сплетение $Z_{q} \prec H^{n}=[K] H^{n}$, где $K-$ база сплетения. Так как $H_{n} / K \cong H^{n} \in \mathfrak{H}$, 


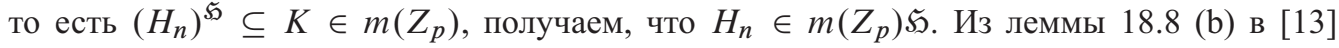
следует, что $O_{p}\left(H_{n}\right)=1$. Поэтому $H_{n} \in f\left(Z_{p}\right)$ в силу леммы 10 . Если $L-$ подгруппа порядка $q$ из $H$, то по лемме 18.2 из [13] группа $Z_{q}$ ? $L^{n}$ изоморфно вкладывается в $H_{n}$. В силу леммы 3.1 .7 из [12], ступень нильпотентности группы $Z_{q}$ 2 $L^{n}$ не меньше $n+1$. Согласно теореме 5 из [4], справедливо равенство $f\left(Z_{p}\right)=\operatorname{form}\left(G / G_{\beta(} Z_{p}\right)$. Обозначим через $\mathfrak{S}_{1}$ формацию, порожденную всеми силовскими $q$-подгруппами из $G / G_{\beta}\left(Z_{p}\right)$. По лемме 3.1 .4 из [12], всякая силовская $q$-подгруппа группы $H_{n}$ принадлежит $\mathfrak{S}_{1}$. Хорошо известно, что формация, порожденная конечным множеством групп, однопорожденна, пусть $\mathfrak{S}_{1}=\operatorname{form} S$ для некоторой группы $S$. Таким образом, $Z_{q}$ ح $L^{n} \in s$ form $S$. Последнее, ввиду леммы 3.1 .5 из [12], означает, что ступень нильпотентности группы $Z_{q} 2 L^{n}$ не превышает (при любом натуральном $n$ ) ступень нильпотентности группы $S$. Получаем противоречие.

Итак, если $p$ и $q$ - различные простые числа и группа $Z_{q}$ принадлежит формации $m\left(Z_{p}\right)$, то $q \notin \pi(\mathfrak{H})$. Более того, как и при доказательстве теоремы 8.18 в [9], можно показать, что в этой ситуации формация $\mathfrak{H}$ абелева. Лемма доказана.

Лемма 12. Пусть $\mathfrak{F}=\mathfrak{M S}-$ однопорожденная $\Omega$-расслоенная формация с направлением $\beta$, причем $\mathfrak{M}=\Omega F(m, \beta)$, где $m-$ внутренний $\Omega$-спутник формации $\mathfrak{M}$, а $\mathfrak{H}-$ неединичная формачия. Тогда если найдется такая группа $Z_{p} \notin \Omega$, что $Z_{p} \in \mathfrak{M}$, то $Z_{p} \notin \mathfrak{H}$. Более того, в этом случае формация $\mathfrak{H}$ абелева.

Доказательство. По условию, для некоторой группы $G$ справедливо равенство $\mathfrak{F}=\Omega F(G, \beta)$. Обозначим через $f$ минимальный $\Omega$-спутник формации $\mathfrak{F}$. Предположим, что $A=Z_{p} \in \mathfrak{H}$. Рассмотрим группу $W=A$ 々 $A^{m}$, где $m=|G|$. Несложно показать, что $W \in \mathfrak{M} \mathfrak{S}=\mathfrak{F}=\Omega F(G, \beta)$. Следовательно, $W \in f\left(\Omega^{\prime}\right)=\operatorname{form}\left(G / O_{\Omega}(G)\right)$. Согласно лемме 3.1 .7 в [12], ступень нильпотентности $W$ не меньше $m+1$, что противоречит лемме 3.1 .5 в [12]. Таким образом, $A \notin \mathfrak{H}$.

Допустим, что $p \in \pi(\mathfrak{S})$. Пусть $H-$ некоторая $p d$-группа из $\mathfrak{H}$. Для всякого натурального числа $n$ через $H_{n}$ обозначим регулярное сплетение $Z_{p} 2 H^{n}=[K] H^{n}$, где $K$ - база сплетения. Так как $H_{n} / K \cong H^{n} \in \mathfrak{S}$, то есть $\left(H_{n}\right)^{\mathfrak{H}} \subseteq K \in \mathfrak{M}$, справедливы включения $H_{n} \in \mathfrak{M} \mathfrak{H}$ и $H_{n} \in f\left(\Omega^{\prime}\right)$. Если $L-$ подгруппа порядка $p$ из $H$, то по лемме 18.2 в [13] группа $Z_{p}<L^{n}$ изоморфно вкладывается в $H_{n}$. Таким образом, $Z_{p} \succ L^{n} \in s$ form $\left(G / O_{\Omega}(G)\right)$. В силу леммы 3.1.7 из [12], ступень нильпотентности группы $Z_{p} \prec L^{n}$ не меньше $n+1$. Последнее противоречит лемме 3.1 .5 из [12].

Так же, как и при доказательстве теоремы 8.18 в [9], можно показать, что в этой ситуации формация $\mathfrak{5}$ абелева. Лемма доказана.

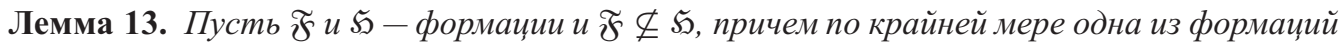
$\mathfrak{F}, \mathfrak{S}$ разрешима. Если $\mathfrak{F}-\Omega$-расслоенная формаџия с направлением $\beta$, то она содержит минимальную $\Omega$-расслоенную не $\mathfrak{H}$-формацию с направлением $\beta$.

Доказательство. Утверждение очевидно при $\mathfrak{H}=\varnothing$. Пусть $\mathfrak{S} \neq \varnothing$ и $G-$ группа минимального порядка из $\mathfrak{F} \backslash \mathfrak{S}$. Тогда $G-$ монолитическая группа с монолитом $R=G^{\mathfrak{H}}$. Покажем, что в $\mathfrak{M}=\Omega F(G, \beta)$ имеется лишь конечное множество $\Omega$-расслоенных подформаций с направлением $\beta$.

Обозначим через $m$ минимальный $\Omega$-спутник формации $\mathfrak{M}$. В силу теоремы 5 из [4], $m(B)=\operatorname{form}\left(G / G_{\beta(B)}\right)$ для любого $B \in K(G) \cap \Omega, m\left(\Omega^{\prime}\right)=\operatorname{form}\left(G / O_{\Omega}(G)\right)$ и $m(B)=\varnothing$, если $B \in \Omega \backslash K(G)$. Если $G_{\beta(B)} \neq 1$, то, ввиду условия леммы, $G / G_{\beta(B)} \in$ ऽ, то есть $\left(G / G_{\beta(B)}\right)^{\varsigma}=1$. Тогда, по теореме 3.47 из [9], в $m(B)$, где $B \in K(G) \cap \Omega$, имеется лишь конечное множество $\Omega$-расслоенных подформаций с направлением $\beta$. Если же $G_{\beta(B)}=1$, 
то $m(B)=\operatorname{form}(G)$ и по лемме 19.6 из [9] $m(B)$ имеет лишь конечное множество подформаций.

Аналогичное рассуждение можно провести для формации $m\left(\Omega^{\prime}\right)$.

Так как множество попарно неизоморфных групп, принадлежащих $K(G) \cap \Omega$, конечно, существует лишь конечное число $\Omega$-спутников $t$ с условием $t \leqslant m$. Из следствия 1 заключаем, что в формации $\mathfrak{M}$ содержится лишь конечное множество $\Omega$-расслоенных подформаций с направлением $\beta$. Так как при этом $\mathfrak{M} \nsubseteq \mathbb{\mathfrak { H }}$, но $\mathfrak{M}_{0} \subseteq \mathfrak{H}$, в $\mathfrak{M}$ можно выбрать такую $\Omega$-расслоенную подформацию $\Omega$ с направлением $\beta$, что $\nwarrow \nsubseteq \mathfrak{H}$, но $\Omega_{1} \subseteq \mathfrak{S}$ для каждой собственной $\Omega$-расслоенной подформации $\Omega_{1}$ с направлением $\beta$ из $\Omega$. Лемма доказана.

Из теоремы 1 в [14] несложно получить следующее утверждение.

Лемма 14. Пусть $\mathfrak{F}$ - минимальная $\Omega$-расслоенная не $\mathfrak{N}_{\omega} \mathfrak{N}_{\omega^{\prime}}$-формация с направлением $\beta$, где $\omega=\pi(\Omega \cap \mathfrak{U})$. Тогда $\mathfrak{F}=\Omega F(G, \beta)$, где $G-$ такая монолитическая группа с

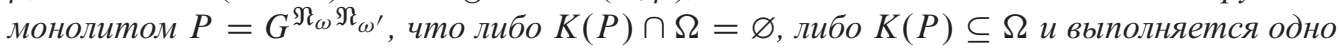
из следующих условии:

(1) $P$ - неабелева группа;

(2) $G=[P] M$, где $P=C_{G}(P)-$-группа и $M=[Q] N$, причем

$$
C_{M}(Q)=Q=M^{\mathfrak{N}_{\omega^{\prime}}}
$$

Лемма 15. Пусть $\mathfrak{F}=\mathfrak{M S}-$ несократимая факторизация однопорожденной $\Omega$-расслоенной формации с направлением $\beta$, где $\mathfrak{M}-$ также $\Omega$-расслоенная формация с направлением $\beta$. Тогда $\mathfrak{M} \subseteq \mathfrak{N}_{\omega} \mathfrak{N}_{\omega^{\prime}}$, где $\omega=\pi(\Omega \cap \mathfrak{U})$.

Доказательство. По условию, для некоторой группы $G$ справедливо равенство $\mathfrak{F}=\Omega F(G, \beta)$. Обозначим через $f$ минимальный $\Omega$-спутник формации $\mathfrak{F}$.

Допустим, что существует простая неабелева группа $T \in \mathfrak{M}$. Для произвольной группы $H$ из $\mathfrak{S}$ рассмотрим регулярное сплетение

$$
W=T ?\left(H^{n}\right)=[K]\left(H^{n}\right),
$$

где $K$ - база сплетения $W$, а $H^{n}$ - прямое произведение $n$ экземпляров группы $H$. По предложению 18.5 в [13], группа $W$ монолитична с монолитом $R=K$. Несложно показать, что $W \in \mathfrak{F}$. Если $T \in \Omega$, то по теореме 5 в [4]

$$
W / W_{\beta(T)} \cong W / O_{T^{\prime}}(W) \cong W \in f(T)=\operatorname{form}\left(G / G_{\beta(T)}\right) .
$$

Ho

$$
|R|=|K|=|T|^{|H|^{n}},
$$

что противоречит лемме 3.1 .5 из [12]. В случае, когда $T \notin \Omega$,

$$
W / O_{\Omega}(W) \cong W \in f\left(\Omega^{\prime}\right)=\operatorname{form}\left(G / O_{\Omega}(G)\right) .
$$

Получаем противоречие с леммой 3.1 .5 из [12]. Следовательно, все простые группы в формации $\mathfrak{M}$ абелевы и $K(\mathfrak{M}) \cap \mathfrak{U} \neq \varnothing$.

Покажем теперь, что $\mathfrak{M} \subseteq \mathfrak{N}_{\omega} \mathfrak{N}_{\omega^{\prime}}$. Предположим, что $\mathfrak{M} \nsubseteq \mathfrak{N}_{\omega} \mathfrak{N}_{\omega^{\prime}}$. Тогда по лемме 13 в $\mathfrak{M}$ имеется минимальная $\Omega$-расслоенная не $\mathfrak{N}_{\omega} \mathfrak{N}_{\omega^{\prime}}$-формация $\mathfrak{M}_{0}$ с направлением $\beta, \mathrm{y}$ 
которой все собственные $\Omega$-расслоенные подформации с направлением $\beta$ содержатся в $\mathfrak{N}_{\omega} \mathfrak{N}_{\omega^{\prime}}$. Причем, по лемме $14, \mathfrak{M}_{0}=\Omega F(Z, \beta)$, где $Z$ - такая монолитическая группа с монолитом $P=Z^{\mathfrak{N}_{\omega} \mathfrak{N}_{\omega^{\prime}}}$, что, либо $K(P) \cap \Omega=\varnothing$, либо $K(P) \subseteq \Omega$ и выполняется одно из следующих условий:

(1) $P$ - неабелева группа;

(2) $Z=[P] M$, где $P=C_{Z}(P)-p$-группа, а $M=[Q] N \neq 1$ и $Q=C_{M}(Q)=M^{\mathfrak{\Re}_{\omega^{\prime}}}$ - монолит группы $M$.

Для всякого натурального числа $n$ через $G_{n}$ обозначим регулярное сплетение

$$
G_{n}=Z 2 H^{n}=[K] H^{n},
$$

где $K$ - база сплетения. Так как $G_{n} / K \cong H^{n} \in \mathfrak{H}$, справедливы включения $\left(G_{n}\right)^{\mathfrak{S}} \subseteq K \in \mathfrak{M}$.

Допустим, что $\left(G_{n}\right)^{\mathfrak{H}}$ входит подпрямо в $K$. Тогда $G_{n}$ принадлежит формации $\mathfrak{F}$. Обозначим $Z_{1}$ первую копию $Z$ в $K$ и $P_{1}$ монолит группы $Z_{1}$. Ввиду локальности $\mathfrak{N}_{\omega} \mathfrak{N}_{\omega^{\prime}}$ заключаем, что $P_{1} \nsubseteq \Phi\left(Z_{1}\right)$. Значит, $P_{1} \nsubseteq Z Z\left(Z_{1}\right)$. Следовательно, по предложению 18.5 в [13], $G_{n}$ монолитична с монолитом

$$
T=\prod_{b \in H^{n}} P_{1}^{b} .
$$

Если $K(P) \cap \Omega=\varnothing$, то $O_{\Omega}(Z)=1$ и $O_{\Omega}\left(G_{n}\right)=1$. Значит, по теореме 5 в [4],

$$
G_{n} / O_{\Omega}\left(G_{n}\right) \cong G_{n} \in f\left(\Omega^{\prime}\right)=\operatorname{form}\left(G / O_{\Omega}(G)\right) .
$$

Но $G_{n}$ содержит монолит порядка $|P|^{|H|^{n}}$, что противоречит лемме 3.1 .5 в [12]. Таким образом, $K(P) \subseteq \Omega$.

Пусть $Z$ удовлетворяет условию 1 и $B \in K(P)$. Очевидно, что $O_{B^{\prime}}(Z)=1$ и $O_{B^{\prime}}\left(G_{n}\right)=1$. По теореме 5 из [4],

$$
G_{n} /\left(G_{n}\right)_{\beta(B)} \cong G_{n} \in f(B)=\operatorname{form}\left(G / O_{B^{\prime}}(G)\right) .
$$

Получаем противоречие с леммой 3.1 .5 в [12].

Пусть $Z$ удовлетворяет условию 2 и $p \in \pi(P)$. Поскольку $P_{1} \nsubseteq \Phi\left(Z_{1}\right)$, ввиду леммы 9.4 в [13], $T \nsubseteq \Phi(K)$ и $T$ дополняема в $G_{n}$. Из монолитичности $G_{n}$ заключаем, что $T$ самоцентрализуема в $G_{n}$. Таким образом, $\left(G_{n}\right)_{\beta\left(Z_{p}\right)}=T$. Поэтому

$$
G_{n} /\left(G_{n}\right)_{\beta\left(Z_{p}\right)} \cong G_{n} / T \cong\left(Z_{1} / P_{1}\right) \prec\left(H^{n}\right) \in f\left(Z_{p}\right) .
$$

Но $Z_{1} / P_{1} \cong M$. Поскольку $Q=C_{M}(Q)$, заключаем, что $Q \nsubseteq Z(M)$, и по предложению 18.5 в [13] группа $\left(Z_{1} / P_{1}\right)$ ? $\left(H^{n}\right)$ имеет минимальную нормальную подгруппу порядка $|Q|^{|H|^{n}}$. Это приводит к противоречию с леммой 3.1 .5 в [12]. Итак, $\left(G_{n}\right)^{\mathfrak{S}}$ не входит подпрямо в $K$.

Пусть $V-$ проекция $\left(G_{n}\right)^{\mathfrak{H}}$ в $Z_{1}$. Поскольку $\left(G_{n}\right)^{\mathfrak{H}}-$ нормальна в $G_{n}$, группа $V$ нормальна в $Z_{1}$. Так как $\left(G_{n}\right)^{\mathfrak{H}}$ не входит подпрямо в $K$, заключаем, что $V \neq Z_{1}$ и, по лемме 3.1 .9 из [12], $\left(Z_{1} / V\right)$ ? $\left(H^{n}\right)$ - гомоморфный образ $G_{n} /\left(G_{n}\right)^{\mathfrak{H}}$. Таким образом,

$$
\left(Z_{1} / V\right) \prec\left(H^{n}\right) \cong G_{n} /(V)^{\#} \in \mathfrak{H} .
$$


Пусть $Z_{1} / X-$ последний фактор главного ряда $Z_{1}$, проходящего через $V$. Тогда $(X)^{\#}-$ нормальная подгруппа в $G_{n}$ и $(V)^{\#} \subseteq(X)^{\#}$. Значит,

$$
G_{n} /(X)^{\#} \cong\left(Z_{1} / X\right) \prec\left(H^{n}\right) \in \mathfrak{H} .
$$

В частности, из этого следует, что $\mathfrak{S}-$ неабелева формация.

Допустим, что найдется такая группа $Z_{p} \notin \Omega$, что $Z_{p} \in \mathfrak{M}$. Тогда, по лемме 12 формация $\mathfrak{5}$ абелева. Получаем противоречие. Как показано выше, каждая простая группа из $\mathfrak{M}$ абелева. Следовательно, все простые группы из $\mathfrak{M}$ являются $\Omega$-группами и $K(\mathfrak{M}) \cap \Omega \cap \mathfrak{U} \neq \varnothing$.

Допустим, что $K(\mathfrak{M}) \cap \Omega \cap \mathfrak{U}=\left(Z_{p}\right)$. Тогда, очевидно, что случай 2 невозможен. Возьмем группу $Y$ из разности $\mathfrak{F} \backslash \mathfrak{S}$, причем минимальную с таким свойством. Тогда $Y$ - монолитическая группа с монолитом $R=Y^{\mathfrak{S}} \in \mathfrak{M}$, где $R-$ элементарная абелева р-группа.

Для произвольного натурального числа $m$ рассмотрим регулярное сплетение

$$
W=Z 2\left(Y^{m} / R^{m}\right),
$$

где $Y^{m}-$ прямое произведение $m$ экземпляров группы $Y$. Допустим, что $W \in \mathfrak{F}$. Если $P$ - неабелева $\Omega$-группа и $(U)=K(P)$, то

$$
W / W_{\beta(U)} \cong W / O_{U^{\prime}}(W) \cong W \in f(U)=\operatorname{form}\left(G / O_{U^{\prime}}(G)\right),
$$

для любого $m \in \mathbf{N}$. Но это противоречит лемме 3.1.5 в [12]. Если $P-\Omega^{\prime}$-группа, то

$$
W / O_{\Omega}(W) \cong W \in f\left(\Omega^{\prime}\right)=\operatorname{form}\left(G / O_{\Omega}(G)\right),
$$

для любого $m \in \mathbf{N}$. Но $P$ не содержится в центре группы $Z$. Следовательно, по предложению 18.5 в [13], $W$ монолитична с монолитом порядка $|P|^{|Y / R|^{m}}$. Получаем противоречие с леммой 3.1 .5 из [12]. Значит, $W \notin \mathfrak{F}$. Поскольку $W / K \in \mathfrak{H}$, где $K-$ база сплетения $W$, справедливы включения $W^{\mathfrak{H}} \subseteq K \in \mathfrak{M}$. Но $W^{\mathfrak{S}}$ не входит подпрямо в $K$. Обозначим через $W_{1}$ проекцию $W^{\mathfrak{H}}$ в $Z_{1}$. Поскольку $W^{\mathfrak{H}}$ нормальна в $W$, заключаем, что $W_{1}$ нормальна в $Z_{1}$. Так как $W^{\mathfrak{H}}$ не входит подпрямо в $K$, заключаем, что $W_{1} \neq Z_{1}$ и, по лемме 3.1 .9 в [12], $\left(Z_{1} / W_{1}\right)$ ᄀ $\left(Y^{m} / R^{m}\right)$ - гомоморфный образ $W / W^{\mathfrak{H}}$. Таким образом,

$$
\left(Z_{1} / W_{1}\right) \prec\left(Y^{m} / R^{m}\right) \cong W /\left(W_{1}\right)^{\#} \in \mathfrak{H} .
$$

Пусть $Z_{1} / X_{1}$ - последний фактор главного ряда $Z_{1}$, проходящего через $W_{1}$. Тогда $\left(X_{1}\right)^{\#}-$ нормальная подгруппа в $W$ и $\left(W_{1}\right)^{\#} \subseteq\left(X_{1}\right)^{\#}$. Значит, $W /\left(X_{1}\right)^{\#} \in \mathfrak{H}$. Поскольку $Z_{1} / X_{1} \in \mathfrak{M}$ и $Z_{1} / X_{1}$ - простая группа, заключаем, что $Z_{1} / X_{1} \cong Z_{p}$ и

$$
W /\left(X_{1}\right)^{\#} \cong Z_{p} 2\left(Y^{m} / R^{m}\right) \in \mathfrak{H} .
$$

Но в этом случае, в силу леммы 3.5.20 из [12],

$$
Y^{m} \in \operatorname{form}\left(Z_{p} \prec\left(Y^{m} / R^{m}\right)\right) \subseteq \mathfrak{H}
$$

и $Y \in \mathfrak{H}$. Получаем противоречие с выбором $Y$. Значит,

$$
|\triangle(K(\mathfrak{M}) \cap \Omega \cap \mathfrak{U})|>1 .
$$

Обозначим $\mathfrak{F}_{1}=\operatorname{form}\left(G / G_{\beta(B)} 0\right.$, где $B \in K(G) \cap \Omega$. Тогда $\mathfrak{F}_{1}=\operatorname{form}(\Gamma)$ для некоторой группы $Г$. Пусть $A_{1}-$ произвольная монолитическая группа из $\mathfrak{S}$. Понятно, что 
$O_{Z_{p}}\left(A_{1}\right)=1$ для некоторого $Z_{p} \in K(\mathfrak{M}) \cap \Omega$. Значит, $A_{1} \in \mathfrak{H} \subseteq m\left(Z_{p}\right) \mathfrak{H}$ и, по лемме 10 , $A_{1} \in f\left(Z_{p}\right)=\operatorname{form}\left(G / G_{\beta\left(Z_{p}\right)}\right) \subseteq \mathfrak{F}_{1}$. Следовательно, всякая монолитическая группа из $\mathfrak{H}$ входит в $\mathfrak{F}_{1}$. Поэтому $\mathfrak{H} \subseteq \mathfrak{F}_{1}$.

Так как $G_{n} /(X)^{\#} \cong\left(Z_{1} / X\right) \curlywedge\left(H^{n}\right) \in \mathfrak{H}$, верно соотношение $\pi\left(Z_{1} / X\right) \cap \pi(\mathfrak{H}) \neq \varnothing$. Поэтому без ограничения общности в качестве $H$ можно взять любую $q d$-группу из $\mathfrak{H}$, где $q \in \pi\left(Z_{1} / X\right) \cap \pi(\mathfrak{S})$. Если $L-$ подгруппа порядка $q$ из $H$, а $T$ - подгруппа порядка $q$ из $Z_{1} / X$, то, в силу леммы 18.2 в [13], группа $T<L^{n}$ изоморфно вкладывается в $\left(Z_{1} / X\right)$ 々 $H^{n}$ и $T \gtrless L^{n} \in s$ form $(\Gamma)$. В силу леммы 3.1 .7 из [12], ступень нильпотентности группы $T \gtrless L^{n}$ не меньше $n+1$. Получаем противоречие с леммой 3.1 .5 из [12].

Остается заключить, что $\mathfrak{M} \subseteq \mathfrak{N}_{\omega} \mathfrak{N}_{\omega^{\prime}}$. Лемма доказана.

Всякое представление формации $\mathfrak{F}$ в виде $\mathfrak{F}=\mathfrak{F}_{1} \ldots \mathfrak{F}_{t}$, где $\mathfrak{F}_{1}, \ldots, \mathfrak{F}_{t}-$ некоторые формации и $\mathfrak{F} \neq \mathfrak{F}_{1} \ldots \mathfrak{F}_{i-1} \mathfrak{F}_{i+1} \ldots \mathfrak{F}_{t}$ при всех $i \in\{1, \ldots, t\}$ называют несократимой факторизацией формации $\mathfrak{F}$. Введем обозначение

$$
\mathfrak{S} / O_{p}(\mathfrak{S})=\operatorname{form}\left(G / O_{p}(G): G \in \mathfrak{S}\right) .
$$

Теорема 1. Пусть $\mathfrak{F}=\mathfrak{M S}-$ несократимая факторизация $\Omega$-расслоенной формации с направлением $\beta$, где $\mathfrak{M}-$ также $\Omega$-расслоенная формация с направлением $\beta$. Тогда и только тогда $\mathfrak{F}$ - однопорожденная $\Omega$-расслоенная формация, когда выполняются следующие условия:

(1) $\mathfrak{M}$ - однопорожденная $\Omega$-расслоенная подформаџия из $\mathfrak{N}_{\omega} \mathfrak{N}_{\omega^{\prime}}$, где $\omega=\pi(\Omega \cap \mathfrak{U})$;

(2) $K(\mathfrak{S}) \cap \Omega \cap \mathfrak{U} \subseteq K(\mathfrak{M}) \cap \Omega$;

(3) $\pi(\mathfrak{M}) \cap \pi(\mathfrak{S}) \subseteq \omega$;

(4) если $\mathfrak{M} \nsubseteq \mathfrak{N}_{\omega}$, то $\mathfrak{H}-$ однопорожденная абелева формация;

(5) если $\mathfrak{M} \subseteq \mathfrak{N}_{\omega} u|\pi(\mathfrak{M})|>1$, то $\mathfrak{H}-$ однопорожденная формация;

(6) если $\mathfrak{M}=\mathfrak{N}_{p}$, где $p \in \omega$, то $\mathfrak{S} / O_{p}(\mathfrak{H})$ - однопорожденная формация.

Доказательство. Обозначим через $f$ минимальный $\Omega$-спутник формации $\mathfrak{F}$.

Докажем необходимость. Пусть $\mathfrak{F}=\Omega F(G, \beta)$ для некоторой группы $G$.

Из леммы 15 следует, что $\mathfrak{M} \subseteq \mathfrak{N}_{\omega} \mathfrak{N}_{\omega^{\prime}}$. По лемме $6, \mathfrak{M}-$ наследственная формация. Значит, $\mathfrak{M} \subseteq \mathfrak{F}$. Тогда по лемме 5 в $\mathfrak{M}$ содержится лишь конечное множество наследственных $\Omega$-расслоенных подформаций с направлением $\beta$. Но, по лемме 6 , любая $\Omega$-расслоенная подформация из $\mathfrak{N}_{\omega} \mathfrak{N}$ наследственна. Таким образом, в $\mathfrak{M}$ существует лишь конечное множество $\Omega$-расслоенных подформаций с направлением $\beta$. Пусть $\mathfrak{M}_{0} \subset \mathfrak{M}_{1} \subset \ldots \subset \mathfrak{M}_{k}=\mathfrak{M}$ - такая цепь $\Omega$-расслоенных формаций с направлением $\beta$, что $\mathfrak{M}_{i-1}-$ максимальная $\Omega$-расслоенная подформация в $\mathfrak{M}_{i}, i=1, \ldots, k$. Пусть $M_{i} \in \mathfrak{M}_{i} \backslash \mathfrak{M}_{i-1}, i=1,2, \ldots, k$. Тогда

$$
\mathfrak{M}=\Omega F\left(M_{1}, \ldots, M_{k}, \beta\right)=\Omega F\left(M_{1} \times \ldots \times M_{k}, \beta\right),
$$

то есть $\mathfrak{M}$ - однопорожденная $\Omega$-расслоенная формация. Справедливо условие 1 .

Допустим, что $K(\mathfrak{S}) \cap \Omega \cap \mathfrak{U} \nsubseteq \mathbb{K}(\mathfrak{M}) \cap \Omega$. Если $\mathfrak{M} \nsubseteq \mathfrak{N}_{\omega}$, найдется $Z_{q} \in \mathfrak{M} \backslash \Omega$. Следовательно, по лемме 12 , формация $\mathfrak{5}$ абелева. Пусть $Z_{p} \in(K(\mathfrak{S}) \cap \Omega) \backslash K(\mathfrak{M})$. Тогда, по лемме $1, \mathfrak{N}_{p} \subseteq \mathfrak{F}$. Несложно показать, что

$$
\mathfrak{N}_{p}=\left(\mathfrak{N}_{p} \cap \mathfrak{M}\right)\left(\mathfrak{N}_{p} \cap \mathfrak{H}\right)=\mathfrak{N}_{p} \cap \mathfrak{H} .
$$


Следовательно, $\mathfrak{N}_{p} \subseteq \mathfrak{S}$. Получаем противоречие с абелевостью формации $\mathfrak{H}$. Значит, $\mathfrak{M} \subseteq \mathfrak{N}_{\omega}$. В этом случае, по лемме 7, в формации $\mathfrak{F}$ содержится бесконечное множество наследственных $\Omega$-расслоенных подформаций с направлением $\beta$, что противоречит лемме 5. Итак, выполняется условие 2.

Допустим, что существует $q \in(\pi(\mathfrak{M}) \cap \pi(\mathfrak{S})) \backslash \omega$. Поскольку формация $\mathfrak{M}$ наследственна и $\mathfrak{M} \subseteq \mathfrak{N}_{\omega} \mathfrak{N}_{\omega^{\prime}}$, найдется такая группа $M \in \mathfrak{M}$, что $|M|=q$. Пусть $H-$ некоторая $q d$-группа из $\mathfrak{H}$. Для любого натурального $n$ рассмотрим регулярное сплетение

$$
D=M 2(H)^{n}=[K](H)^{n},
$$

где $K$ - база сплетения. Если $L-$ подгруппа порядка $q$ из $H$, то по лемме 18.2 из [13] группа $M>(L)^{n}$ изоморфно вкладывается в $M<(H)^{n}$. Несложно показать, что $D$ принадлежит $\mathfrak{F}$. Но в этом случае

$$
D / O_{\Omega}(D) \cong D \in f\left(\Omega^{\prime}\right)=\operatorname{form}\left(G / O_{\Omega}(G)\right), \quad M \imath(L)^{n} \in s \text { form }\left(G / O_{\Omega}(G)\right) .
$$

Получили противоречие с леммой 3.1 .5 в [12]. Таким образом, справедливо условие 3.

Как отмечено выше, если $\mathfrak{M} \nsubseteq \mathfrak{N}_{\omega}$, то формация $\mathfrak{5}$ абелева. Очевидно, что существует группа $M \in \mathfrak{M}$ такая, что $|M|=q \notin \omega$. Пусть $H-$ произвольная группа из $\mathfrak{H}$. Для произвольного натурального $n$ рассмотрим регулярное сплетение

$$
D=M\left((H)^{n}=[K](H)^{n},\right.
$$

где $K$ - база сплетения. Несложно показать, что $D$ принадлежит $\mathfrak{F}$. Но в этом случае

$$
D / O_{\Omega}(D) \cong D \in f\left(\Omega^{\prime}\right)=\operatorname{form}\left(G / O_{\Omega}(G)\right)
$$

и $H \in f\left(\Omega^{\prime}\right)$. Следовательно, $\mathfrak{H} \subseteq f\left(\Omega^{\prime}\right)$. Тогда, в силу леммы 3.1 .5 в [12], число неизоморфных монолитических групп из $\mathfrak{5}$ конечно. Значит, в этом случае формация $\mathfrak{S}$ однопорожденна.

Предположим теперь, что $\mathfrak{M} \subseteq \mathfrak{N}_{\omega}$ и $|\pi(\mathfrak{M})|>1$. Обозначим $\mathfrak{F}_{1}=\operatorname{form}\left(G / G_{\beta(B)}\right.$, где $B \in K(G) \cap \Omega)$. Тогда $\mathfrak{F}_{1}=\operatorname{form}(\Gamma)$ для некоторой группы Г. Пусть $A_{1}-$ произвольная монолитическая группа из $\mathfrak{5}$. Понятно, что $O_{Z_{p}}\left(A_{1}\right)=1$ для некоторого $Z_{p} \in K(\mathfrak{M}) \cap \Omega$. Значит, $A_{1} \in \mathfrak{S} \subseteq m\left(Z_{p}\right) \mathfrak{H}$ и, по лемме $10, A_{1} \in f\left(Z_{p}\right)=\operatorname{form}\left(G / G_{\beta\left(Z_{p}\right)}\right) \subseteq \mathfrak{F}_{1}$. Следовательно, всякая монолитическая группа из $\mathfrak{H}$ входит в $\mathfrak{F} 1$. Поэтому $\mathfrak{H} \subseteq \mathfrak{F}_{1}$.

Пусть $\mathfrak{H} \subseteq \mathfrak{A}$. Тогда, в силу леммы 3.1 .5 из [12], число неизоморфных монолитических групп из $\mathfrak{5}$ конечно. Значит, формация $\mathfrak{S}$ однопорожденна. Пусть $\mathfrak{S} \nsubseteq \mathbb{A}$. Поскольку для минимального $\Omega$-спутника $m$ формации $\mathfrak{M}$ справедливо равенство $m\left(Z_{p}\right)=$ (5 при всех $Z_{p} \in K(\mathfrak{M}) \cap \Omega$, равенства $h\left(Z_{p}\right)=m\left(Z_{p}\right) \mathfrak{H}=\mathfrak{H}$ и $h(B)=\mathfrak{H}$ справедливы для $B \in(K(\mathfrak{S}) \cap \Omega) \backslash K(\mathfrak{M})$. Но $f \leqslant h$. Значит, $\mathfrak{F}_{1} \subseteq \mathfrak{H}$. Заключаем, что $\mathfrak{H}=\mathfrak{F}_{1}-$ однопорожденная формация.

Возьмем теперь $\mathfrak{M}=\mathfrak{N}_{p}$. Покажем, что в этом случае $\mathfrak{F}=\mathfrak{N}_{p} \mathfrak{H}=\mathfrak{N}_{p}\left(\mathfrak{H} / O_{p}(\mathfrak{H})\right)$. Очевидно, что $\mathfrak{N}_{p}\left(\mathfrak{H} / O_{p}(\mathfrak{H})\right) \subseteq \mathfrak{N}_{p} \mathfrak{H}$. Пусть теперь $N \in \mathfrak{N}_{p} \mathfrak{H}$. Тогда $N^{\mathfrak{H}} \in \mathfrak{N}_{p}$, то есть $N^{\mathfrak{H}} \subseteq O_{p}(N)$. Значит, $N / O_{p}(N) \in \mathfrak{H}$. Но $O_{p}\left(N / O_{p}(N)\right)=1$, и следовательно, $N / O_{p}(N) \in \mathfrak{S} / O_{p}(\mathfrak{S})$. Поэтому $N \in \mathfrak{N}_{p}\left(\mathfrak{S} / O_{p}(\mathfrak{S})\right)$ и $\mathfrak{N}_{p} \mathfrak{H} \subseteq \mathfrak{N}_{p}\left(\mathfrak{S} / O_{p}(\mathfrak{S})\right)$. В силу леммы $8, \mathfrak{F}$ имеет $\Omega$-спутник $f_{1}$ такой, что $f_{1}\left(Z_{p}\right)=\mathfrak{H} / O_{p}(\mathfrak{H})$. Пусть $H-$ произвольная группа из $\mathfrak{S}$, тогда $H / O_{p}(H) \in \mathfrak{S} / O_{p}(\mathfrak{S})=f_{1}\left(Z_{p}\right)$. Поскольку $O_{p}\left(H / O_{p}(H)\right)=1$, по лемме $10, H / O_{p}(H) \in f\left(Z_{p}\right) \subseteq \mathfrak{F}_{1}$. Следовательно, $\mathfrak{S} / O_{p}(\mathfrak{S}) \subseteq \mathfrak{F} 1$. По определению $f \leqslant f_{1}$. Поэтому $\mathfrak{F}_{1} \subseteq \mathfrak{S} / O_{p}(\mathfrak{S})$ и $\mathfrak{F}_{1}=\mathfrak{S} / O_{p}(\mathfrak{S})-$ однопорожденная формация.

Докажем достаточность. Если $K(\mathfrak{F}) \cap \Omega=\varnothing$, то $\mathfrak{M} \subseteq \mathfrak{N}_{\omega^{\prime}}$. Тогда $\mathfrak{M}=\Omega F(M, \beta)$ и, по лемме 3 из [4], $\mathfrak{M}=\operatorname{form}(M)$. Значит, в силу следствия 3.3.3 в [12], ₹ - однопорожденная 
формация и, в силу леммы 3 в [4], $\mathfrak{F}$ - однопорожденная $\Omega$-расслоенная формация с направлением $\beta$.

Пусть $K(\mathfrak{F}) \cap \Omega \neq \varnothing$ и $\mathfrak{M} \nsubseteq \mathfrak{N}_{\omega}$. Заметим, что формация $\mathfrak{F}$ разрешима. Из п. 2 следует, что $K(\mathfrak{F}) \cap \Omega=K(\mathfrak{M}) \cap \Omega$. Поскольку $\mathfrak{M}$ - однопорожденная $\Omega$-расслоенная формация, по лемме $9, \triangle(\mathfrak{M}) \cap \Omega-$ конечное множество. В силу леммы 8 , формация $\mathfrak{F}$ имеет такой внутренний $\Omega$-спутник $t$, что $t(A)=m(A) \mathfrak{H}$, если $A \in K(\mathfrak{M}) \cap \Omega$ и $t\left(\Omega^{\prime}\right)=m\left(\Omega^{\prime}\right) \mathfrak{H}$.

По условию $\mathfrak{M}$ - однопорожденная $\Omega$-расслоенная формация и $\mathfrak{M} \subseteq \mathfrak{N}_{\omega} \mathfrak{N}_{\omega^{\prime}}$. Кроме того, в силу п. $3, \pi(\mathfrak{M}) \cap \pi(\mathfrak{S}) \subseteq \omega$. Следовательно, $\pi(m(A)) \cap \pi(\mathfrak{S})=\varnothing$ для любого $A K(\mathfrak{M}) \cap \Omega$ и $\pi\left(m\left(\Omega^{\prime}\right)\right) \cap \pi(\mathfrak{H})=\varnothing$. Значит, в силу следствия 3.3 .3 в [12], $m(A) \mathfrak{H}$ и $m\left(\Omega^{\prime}\right) \mathfrak{H}$ однопорожденны.

Таким образом, по лемме $9, \mathfrak{F}$ является однопорожденной $\Omega$-расслоенной формацией с направлением $\beta$.

Допустим, что $\mathfrak{M} \subseteq \mathfrak{N}_{\omega}$ и $|\pi(\mathfrak{M})|>1$. В силу леммы 8, формация $\mathfrak{F}$ имеет такой расслоенный $\Omega$-спутник $f$, что $f(C)=\mathfrak{H}$ для всех $C \in K(\mathfrak{F}) \cap \Omega$ и $f\left(\Omega^{\prime}\right)=\mathfrak{H}$. По условию $1, \mathfrak{M}$ - однопорожденная $\Omega$-расслоенная формация. Значит, по лемме 9 , $\triangle(\mathfrak{M}) \cap \Omega-$ конечное множество. Из теоремы 1 в [4] и теоремы 2 в [4] следует, что $\mathfrak{S}$ является свободной формацией. Тогда из теоремы 5 в [4] несложно получить, что $\triangle(\mathfrak{S})$ - конечное множество. Очевидно, что $K(\mathfrak{F}) \subseteq K(\mathfrak{S}) \cup K(\mathfrak{M})$. Поэтому $\triangle(\mathfrak{F}) \cap \Omega-$ конечное множество. Следовательно, в силу леммы $9, \mathfrak{F}-$ однопорожденная $\Omega$-расслоенная формация с направлением $\beta$.

Пусть $\mathfrak{N}_{p}=\mathfrak{M}$ и $\mathfrak{F}=\mathfrak{N}_{p} \mathfrak{H}$. Как показано ранее, $\mathfrak{F}=\mathfrak{N}_{p} \mathfrak{H}=\mathfrak{N}_{p}\left(\mathfrak{H} / O_{p}(\mathfrak{H})\right)$. По лемме $8, \mathfrak{F}$ имеет $\Omega$-спутник $f_{1}$ такой, что $f_{1}(C)=\mathfrak{5} / O_{p}(\mathfrak{5})$ для всех $C \in K(\mathfrak{F}) \cap \Omega$ и $f_{1}\left(\Omega^{\prime}\right)=\mathfrak{S} / O_{p}(\mathfrak{S})$. Из теорем 1 в [4] и 2 в [4] следует, что $\mathfrak{S} / O_{p}(\mathfrak{S})$ является свободной формацией. Тогда с учетом теоремы 5 из [4] легко показать, что $\triangle\left(\mathfrak{H} / O_{p}(\mathfrak{S})\right)$ - конечное множество. Но в этом случае $\triangle(\mathfrak{F})$ - конечное множество. Следовательно, по лемме 9 , $\mathfrak{F}$ - однопорожденная $\Omega$-расслоенная формация с направлением $\beta$. Теорема доказана.

Теорема 2. Пусть $\mathfrak{F}=\mathfrak{M}_{1} \ldots \mathfrak{M}_{t}-$ несократимая факторизация $\Omega$-расслоенной формации $\mathfrak{F}$ с направлением $\beta$, причем формачия $\mathfrak{M}_{1}$ также является $\Omega$-расслоенной $c$ направлением $\beta$. Формачия $\mathfrak{F}$ тогда и только тогда однопорожденна, когда выполняются условия:

(1) $t \leqslant 3, K(\mathfrak{F}) \cap \mathfrak{U} \cap \Omega=K\left(\mathfrak{M}_{1}\right) \cap \mathfrak{U} \cap \Omega$;

(2) при $t=2$ выполняются все пункты теоремы 1;

(3) если $t=3$, то $\mathfrak{M}_{1}$ и $\mathfrak{M}_{1} \mathfrak{M}_{2}$ - однопорожденные $\Omega$-расслоенные формации с направлением $\beta$, $\mathfrak{M}_{2}$, $\mathfrak{M}_{3}$ однопорожденны, причем $\mathfrak{M}_{1} \subseteq \mathfrak{N}_{\omega}$, $\mathfrak{M}_{2} \subseteq \mathfrak{N}_{\omega^{\prime}}$, $\mathfrak{M}_{3}$ абелева и $\pi\left(\mathfrak{M}_{1} \mathfrak{M}_{2}\right) \cap \pi\left(\mathfrak{M}_{3}\right) \subseteq \omega$.

Доказательство. Докажем необходимость. Пусть $\mathfrak{H}_{1}=\mathfrak{M}_{2} \ldots \mathfrak{M}_{t}$, то есть $\mathfrak{F}=\mathfrak{M}_{1} \mathfrak{H}_{1}$, и $\mathfrak{F}=\Omega F(G, \beta)$ для некоторой группы $G$. По лемме $15, \mathfrak{M}_{1} \subseteq \mathfrak{N}_{\omega} \mathfrak{N}_{\omega^{\prime}}$.

Допустим, что $K(\mathfrak{F}) \cap \Omega=\varnothing$. Тогда $\mathfrak{M}_{1} \subseteq \mathfrak{N}_{\omega^{\prime}}$. Следовательно, по лемме $12, \mathfrak{H}_{1}-$ абелева формация. Учитывая лемму 3.2 .5 из [12], видим, что $t=2$ и теорема является частным случаем теоремы 1.

Пусть $K(\mathfrak{F}) \cap \Omega \neq \varnothing$. Для любого $Z_{p} \in K(\mathfrak{F}) \cap \Omega$ выполняются равенства

$$
\mathfrak{N}_{p}=\mathfrak{N}_{p} \cap \mathfrak{F}=\left(\mathfrak{N}_{p} \cap \mathfrak{M}_{1}\right)\left(\mathfrak{N}_{p} \cap \mathfrak{H}_{1}\right) .
$$

В силу следствия 3.3 .7 в [12], формации $\mathfrak{N}_{p} \cap \mathfrak{M}_{1}$ и $\mathfrak{N}_{p} \cap \mathfrak{H}_{1}$ не могут одновременно быть отличны от $\mathfrak{M}_{0}$ и $\mathfrak{N}_{p}$. Поэтому либо $\mathfrak{N}_{p} \subseteq \mathfrak{M}_{1}$, либо $\mathfrak{N}_{p} \subseteq \mathfrak{H}_{1}$. Допустим, что 
найдется $\mathfrak{N}_{p} \subseteq \mathfrak{H}_{1}$ и $\mathfrak{N}_{p} \not \mathfrak{M}_{1}$. Если $\mathfrak{M}_{1} \nsubseteq \mathfrak{N}_{\omega}$, то найдется $Z_{q} \in \mathfrak{M}_{1} \backslash \Omega$. Следовательно, по лемме 12 , формация $\mathfrak{H}_{1}$ абелева. Получаем противоречие. Значит, $\mathfrak{M}_{1} \subseteq \mathfrak{N}_{\omega}$. В этом случае, в силу леммы 7 , в формации $\mathfrak{F}$ содержится бесконечное множество наследственных $\Omega$-расслоенных подформаций с направлением $\beta$, что противоречит лемме 5 . Следовательно,

$$
K(\mathfrak{F}) \cap \mathfrak{U} \cap \Omega=K\left(\mathfrak{M}_{1}\right) \cap \mathfrak{U} \cap \Omega
$$

Пусть $t>2$. Введем обозначения $\mathfrak{M}=\mathfrak{M}_{1} \mathfrak{M}_{2}$ и $\mathfrak{H}=\mathfrak{M}_{3} \ldots \mathfrak{M}_{t}$. Допустим, что существует $A \in\left(K\left(\mathfrak{M}_{2}\right) \cap \mathfrak{U} \cap \Omega\right) \backslash K(\mathfrak{F})$. Тогда найдется группа $M \in \mathfrak{M}_{2}$ такая, что $A \in K(M) \cap \Omega$. Для произвольной группы $H \in \mathfrak{H}$ рассмотрим регулярное сплетение

$$
V=M \imath H=[K] H,
$$

где $K$ - база сплетения $V$. Очевидно, что $V^{\mathfrak{S}} \subseteq K \in \mathfrak{M}_{2} \subseteq \mathfrak{M}_{1} \mathfrak{M}_{2}$. Поскольку $K(M) \nsubseteq K(\mathfrak{F})$, ни $V \notin \mathfrak{F}$, ни $V^{\mathfrak{S}}$ не входят подпрямо в $K$ для произвольной группы $H$ из $\mathfrak{5}$. В силу леммы 3.1 .9 в [12], группе $H$ можно сопоставить такую собственную нормальную подгруппу $M_{H}$ группы $M$, что $\left(M / M_{H}\right)$ 乙 $H$ - гомоморфный образ группы $V / V^{\mathfrak{S}} \in \mathfrak{H}$.

Зафиксируем теперь некоторую неединичную группу $H \in \mathfrak{S}$ и рассмотрим бесконечную последовательность $V_{1}=\left(M / M_{H}\right)<H, V_{2}=\left(M / M_{V_{1}}\right)<V_{1}, \ldots$, $V_{t}=\left(M / M_{V_{t-1}}\right) 2 V_{t-1}, \ldots$ Причем, как отмечалось выше, $V_{i} \in \mathfrak{H}$ для всех натуральных $i$. Несложно показать, что все простые группы из $\mathfrak{M}_{1} \mathfrak{M}_{2}$ абелевы. Тогда найдется такое простое число $p$ и такая бесконечная последовательность индексов $j_{1}, j_{2}, \ldots, j_{n}, \ldots$, что $Z_{p} \in K\left(M / M_{V_{j_{k}}}\right)$ при любом $k=1,2, \ldots$ Пусть $T_{1}=Z_{p}, T_{2}=Z_{p} 2 T_{1}, \ldots$, $T_{n+1}=Z_{p} 2 T_{n}, \ldots$ Рассуждая, как и в доказательстве предложения 3.5.21 в [12], можно показать, что для любого натурального числа $m$ найдется такое натуральное число $r$, что $T_{m}$ будет изоморфна некоторой подгруппе $V_{r}$ и $\mathfrak{N}_{p} \subseteq S(\mathfrak{H})$. Таким образом, для любого натурального числа $n$ в $\mathfrak{H}$ существует такая группа $T_{n}$, у которой одна из ее подгрупп является циклической группой порядка $p^{n}$ и $O_{p^{\prime}}\left(T_{n}\right)=1$.

Предположим, что существует группа $Z_{q} \in K(\mathfrak{F}) \cap \Omega, q \neq p$. Тогда $\mathfrak{N}_{q} \subseteq \mathfrak{M}_{1}$. Рассмотрим сплетение $D=Z_{q}$ 々 $T_{n}$. Ясно, что $D \in \mathfrak{F}$ и $D / D_{\beta\left(Z_{q}\right)} \cong T_{n} \in f\left(Z_{q}\right)$ - однопорожденная формация. Получили противоречие с леммой 3.1 .5 в [12]. Следовательно, $Z_{p} \in \Omega, K(\mathfrak{F}) \cap \mathfrak{U} \cap \Omega=\left(Z_{p}\right)$ б и $\mathfrak{M}_{1}=\mathfrak{N}_{p}$.

Допустим, что найдется такая группа $Z_{q} \notin \Omega$, что $Z_{q} \in \mathfrak{M}_{1} \mathfrak{M}_{2}$. Тогда по лемме 12 формация $\mathfrak{H}$ абелева. Получаем противоречие с тем, что $\mathfrak{N}_{p} \subseteq s$ form $\mathfrak{H}$. Поскольку каждая простая группа из $\mathfrak{M}_{1} \mathfrak{M}_{2}$ абелева, все простые группы из $\mathfrak{M}_{1} \mathfrak{M}_{2}$ являются $\Omega$-группами.

Пусть $\mathfrak{N}_{p} \mathfrak{H} \nsubseteq \mathfrak{H}$ и группа $U$ - минимальная группа из разности $\mathfrak{N}_{p} \mathfrak{H} \backslash \mathfrak{H}$. Тогда $U-$ монолитическая группа с монолитом $R=U^{\mathfrak{H}} \in \mathfrak{N}_{p}$. Рассмотрим регулярное сплетение

$$
V=M \imath(U / R)=[K](U / R),
$$

где $K$ - база сплетения $V$. Как показано выше, $V \notin \mathfrak{F}$ и $V^{\mathfrak{5}}$ не входит подпрямо в $K$. Обозначим через $M_{1}$ первую копию $M$ в $K$ и через $V_{1}$ проекцию $V^{\mathfrak{5}}$ в $M_{1}$. Поскольку $V^{\mathfrak{5}}$ нормальна в $V, V_{1}$ также нормальна в $M_{1}$. Так как $V^{\mathfrak{5}}$ не входит подпрямо в $K$, справедливо соотношение $V_{1} \neq M_{1}$ и, по лемме 3.1 .9 из [12], $\left(M_{1} / V_{1}\right)$ ᄀ $(U / R)$ - гомоморфный образ $V / V^{\mathfrak{H}}$. Таким образом,

$$
\left(M_{1} / V_{1}\right) \zeta(U / R) \cong V /\left(V_{1}\right)^{\#} \in \mathfrak{H} .
$$


Пусть $M_{1} / X_{1}$ - последний фактор главного ряда $M_{1}$, проходящего через $V_{1}$. Тогда $\left(X_{1}\right)^{\#}-$ нормальная подгруппа в $V$ и $\left(V_{1}\right)^{\#} \subseteq\left(X_{1}\right)^{\#}$. Значит, $V /\left(X_{1}\right)^{\#} \in \mathfrak{H}$. Поскольку $M_{1} / X_{1} \in \mathfrak{M}_{2}$ и $M_{1} / X_{1}-$ простая группа, справедливы соотношения

$$
M_{1} / X_{1}=Z_{p}, \quad V /\left(X_{1}\right)^{\#} \cong Z_{p} 2(U / R) \in \mathfrak{H} .
$$

Но в этом случае, по лемме 3.5 .20 в [12], $U \in \operatorname{form}\left(Z_{p} \imath(U / R)\right) \subseteq \mathfrak{S}$ и $U \in \mathfrak{S}$. Получили противоречие с выбором $U$. Значит, $\mathfrak{N}_{p} \mathfrak{H}=\mathfrak{H}$.

Возьмем группу $Y$ из разности $\mathfrak{F} \backslash \mathfrak{H}$, причем минимального порядка с таким свойством. Тогда $Y-$ монолитическая группа с монолитом $R=Y^{\mathfrak{H}} \in \mathfrak{M}_{1} \mathfrak{M}_{2}$. Как сказано выше, каждая простая группа из $\mathfrak{M}_{1} \mathfrak{M}_{2}$ - абелева $\Omega$-группа. Значит, $R-$ элементарная абелева $p$-группа и $Y \in \mathfrak{N}_{p} \mathfrak{H}=\mathfrak{S}$. Получаем противоречие с выбором группы $Y$.

Итак, остается заключить, что

$$
K\left(\mathfrak{M}_{2}\right) \cap \mathfrak{U} \cap \Omega \subseteq K(\mathfrak{F}) \cap \mathfrak{U} \cap \Omega \subseteq K\left(\mathfrak{M}_{1}\right)
$$

По лемме 8, $\mathfrak{M}_{1} \mathfrak{M}_{2}-\Omega$-расслоенная формация с направлением $\beta$. Следовательно, по теореме $1, \mathfrak{M}_{1}$ и $\mathfrak{M}_{1} \mathfrak{M}_{2}$ - однопорожденные $\Omega$-расслоенные формации с направлением $\beta$, содержащиеся в $\mathfrak{N}_{\omega} \mathfrak{N}_{\omega^{\prime}}$. Кроме того, так как $\left|\pi\left(\mathfrak{M}_{1} \mathfrak{M}_{2}\right)\right|>1$, по лемме 3.2 .5 в [12] $\mathfrak{M}_{1} \mathfrak{M}_{2}$ не нильпотентна. Значит, по теореме $1, \mathfrak{H}-$ однопорожденная абелева формация и, используя лемму 3.2.5 из [12], заключаем, что $t=3$, причем $\pi\left(\mathfrak{M}_{1} \mathfrak{M}_{2}\right) \cap \pi\left(\mathfrak{M}_{3}\right) \subseteq \omega$.

Утверждение 2 непосредственно следует из теоремы 1.

Пусть $t=3$. Допустим, что $\mathfrak{M}_{1} \nsubseteq \mathfrak{N}_{\omega}$. Тогда, по теореме $1, \mathfrak{M}_{2} \mathfrak{M}_{3}$ - абелева формация. В этом случае $\mathfrak{M}_{2} \mathfrak{M}_{3}$ ограничена по параметру $c$, что противоречит лемме 3.2 .5 в [12]. Следовательно, $\mathfrak{M}_{1} \subseteq \mathfrak{N}_{\omega}$.

Покажем, что $\mathfrak{M}_{2}-$ нильпотентная формация. Пусть $m_{1}-$ минимальный $\Omega$-спутник формации $\mathfrak{M}_{1} \mathfrak{M}_{2}$. По теореме 5 из [4], $m_{1}\left(Z_{p}\right) \subseteq \mathfrak{N}_{\omega^{\prime}}$ для всех $Z_{p} \in K\left(\mathfrak{M}_{1} \mathfrak{M}_{2}\right) \cap \Omega$. Пусть $\left|\pi\left(\mathfrak{M}_{1} \cap \Omega\right)\right|>1$. Введем обозначение

$$
\mathfrak{X}=\bigcup_{p \in \pi\left(\mathfrak{M}_{1}\right)} m_{1}\left(Z_{p}\right) .
$$

Предположим, что $\mathfrak{M}_{2} \not R_{0}(\mathfrak{X})$. Пусть $A-$ группа минимального порядка из $\mathfrak{M}_{2} \backslash R_{0}(\mathfrak{X})$. Тогда $A-$ монолитическая группа с монолитом $R$. Поскольку $\mathfrak{M}_{1} \mathfrak{M}_{2}-$ метанильпотентная формация, $R$ - элементарная абелева $p$-группа для некоторого простого числа $p$. Пусть $q \in \pi\left(\mathfrak{M}_{1}\right) \backslash\{p\}$ и $T=Z_{q} \prec A$. Поскольку $O_{q}(A)=1$, в силу леммы 2 в [11], $T_{\gamma\left(Z_{q}\right)}=K$, где $K-$ база регулярного сплетения $T$. Кроме того, $T_{\alpha\left(Z_{q}\right)}=K$. Но $T / K \cong A \in \mathfrak{M}_{2}$. Поэтому $T \in \mathfrak{N}_{q} \mathfrak{M}_{2} \subseteq \mathfrak{M}_{1} \mathfrak{M}_{2}$. Следовательно,

$$
T / T_{\beta\left(Z_{q}\right)}=T / K \cong A \in m_{1}\left(Z_{q}\right) .
$$

Получаем противоречие. Значит, $\mathfrak{M}_{2} \subseteq R_{0}(\mathfrak{X}) \subseteq \mathfrak{N}_{\omega^{\prime}}$.

Пусть $\mathfrak{M}_{1}=\mathfrak{N}_{p}$ и $\mathfrak{M}_{2} \subseteq \mathfrak{N}_{p} \mathfrak{N}_{\omega^{\prime}}$. Как и в доказательстве теоремы 1 , несложно показать, что

$$
\mathfrak{M}_{p} \mathfrak{M}_{2}=\mathfrak{N}_{p}\left(\mathfrak{M}_{2} / O_{p}\left(\mathfrak{M}_{2}\right)\right)
$$

причем $\mathfrak{M}_{2} / O_{p}\left(\mathfrak{M}_{2}\right) \subseteq \mathfrak{N}_{\omega^{\prime}}$. Таким образом, вместо формации $\mathfrak{M}_{2}$ можно взять $\mathfrak{M}_{2} / O_{p}\left(\mathfrak{M}_{2}\right)$.

Из этого следует, что в любом случае, по теореме 1 , формация $\mathfrak{M}_{2}$ однопорожденна. Необходимость доказана.

Достаточность следует из теоремы 1. Теорема доказана. 
Лемма 16. Пусть

$$
\mathfrak{F}=\mathfrak{M}_{1} \mathfrak{H}_{1}=\mathfrak{M}_{2} \mathfrak{H}_{2}
$$

- несократимая факторизация однопорожденной $\Omega$-расслоенной формации $\mathfrak{F}$ с направлением $\beta$, причем формации $\mathfrak{M}_{1}$ и $\mathfrak{M}_{2}$ также являются $\Omega$-расслоенными с направлением $\beta$. Если $\mathfrak{M}_{1} \nsubseteq \mathfrak{N}$ и $\mathfrak{M}_{2} \nsubseteq \mathfrak{N}$, то $\mathfrak{H}_{1}=\mathfrak{S}_{2}$.

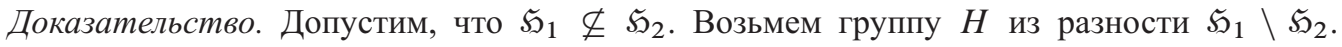
Поскольку $\mathfrak{M}_{1} \nsubseteq \mathfrak{N}$, по лемме 13 в $\mathfrak{M}_{1}$ существует минимальная $\Omega$-расслоенная не $\mathfrak{N - ф о р м а ц и я ~} \mathfrak{M}_{0}$ с направлением $\beta$. В силу теоремы 1 из [14], $\mathfrak{M}_{0}=\Omega F(G, \beta)$, где $G$ - такая монолитическая группа с монолитом $P=G^{\mathfrak{N}}$, что либо $K(P) \cap \Omega=\varnothing$, либо $K(P) \subseteq \Omega$ и $G=[P] M$, где $P=C_{G}(P)-p$-группа, а $M \neq 1-$ монолитическая группа с монолитом $Q=M^{h(A)}$ для $A \in K(P)$, где $h$ - максимальный внутренний $\Omega$-спутник формации $\Re$.

По лемме $15, G \in \mathfrak{M}_{1} \subseteq \mathfrak{N}_{\omega} \mathfrak{N}_{\omega^{\prime}}$, где $\omega=\pi(\Omega \cap \mathfrak{U})$, но $G \notin \mathfrak{N}$. Значит, $G^{\mathfrak{N}_{\omega^{\prime}}} \neq 1$ и $G^{\mathfrak{N}_{\omega^{\prime}}} \in \mathfrak{N}_{\omega}$. Следовательно, $P \subseteq \mathfrak{N}_{\omega}$ и $K(P) \subseteq \Omega$.

Ясно, что $h(A)=\mathfrak{N}_{p}$ для $A \in K(P)$ и $M / Q \in \mathfrak{N}_{p}$, но

$$
O_{p}(M)=O_{p}(G / P)=O_{p}\left(G / C_{G}(P)\right)=1 .
$$

Следовательно, $Q=M \cong Z_{q}, q \neq p$. Обозначим через $W$ регулярное сплетение

$$
W=G \prec H=[K] H,
$$

где $K-$ база сплетения $W$. Если $Z(G) \neq 1$, то $P \subseteq Z(G)$ и $M \triangleleft G$. Получаем противоречие. Таким образом, $Z(G)=1$ и $P \not Z Z(G)$. Тогда, по лемме 3.1 .9 в [12], $W-$ монолитическая группа с монолитом

$$
L=\prod_{b \in H} P^{b} .
$$

Из насыщенности формации $\mathfrak{\Re}$ следует, что $P \nsubseteq \Phi(G)$. Поэтому $L \nsubseteq \Phi(K)$ и $D-$ дополнение к $L$ в $K$, а $D H-$ дополнение к $L$ в $W$. Из монолитичности $W$ следует, что $C_{W}(L)=L$, а значит, $F(W)=L$.

Поскольку $W / K \cong H \in \mathfrak{H}_{1}$, справедливо включение $W^{\mathfrak{H}_{1}} \subseteq K$. Так как $G \in \mathfrak{M}_{1}$ и $K(P) \subseteq \Omega$, справедливы соотношения

$$
G / G_{\beta\left(Z_{p}\right)} \cong G / P \cong M \cong Z_{q} \in m\left(Z_{p}\right),
$$

где $m$ - внутренний $\Omega$-спутник формации $\mathfrak{M}_{1}$. Следовательно, по лемме $11, \mathfrak{S}_{1}-$ абелева формация и $Z_{q} \notin \mathfrak{H}_{1}$. По лемме 2.4 в [10], $\mathfrak{H}_{1}-$ наследственная формация и $q \notin \pi\left(\mathfrak{H}_{1}\right)$. Ясно, что $W^{\mathfrak{H}_{1}} \neq 1$. Допустим, что $W^{\mathfrak{H}_{1}} \subset K$, тогда $K / W^{\mathfrak{H}_{1}}-q$-группа и $W / W^{\mathfrak{H}_{1}}-$ $q d$-группа. Получаем противоречие. Значит, $W^{\mathfrak{H}_{1}}=K \in \mathfrak{M}_{1}$ и $W \in \mathfrak{M}_{1} \mathfrak{H}_{1}=\mathfrak{M}_{2} \mathfrak{H}_{2}$.

Покажем, что $K-\mathfrak{N}^{2}$-радикал группы $W$. Пусть $T-$ нормальная метанильпотентная подгруппа группы $W$. Очевидно, что $F(T) \triangleleft W$ и $F(T) \subseteq F(W)=L$. Если $F(T)=1$, то $T=1$ и $T \subseteq K$. Допустим, что $F(T)=L$. Рассмотрим регулярное сплетение

$$
R=(G / P) \curlywedge H \cong Z_{q} \prec H=\left[K_{1}\right] H,
$$

где $K_{1}-$ база сплетения $R$. Поскольку $q \notin \pi\left(\mathfrak{S}_{1}\right)$, справедливо равенство $O_{q}(H)=1$ и, по лемме 2 из [11], $F_{Z_{q}}(R)=K_{1}$. Значит, $F(R)=K_{1}$. Тогда $F(R) \cong K / L-$ наибольшая 
нильпотентная подгруппа из $W / L$. Таким образом, $T / L \subseteq K / L$ и $T \subseteq K$. Итак, $K-$ $\mathfrak{N}^{2}$-радикал группы $W$.

Ранее доказано, что $W^{\mathfrak{H}_{2}} \in \mathfrak{M}_{2} \subseteq \mathfrak{N}_{\omega} \mathfrak{N}_{\omega^{\prime}}$, поэтому $W^{\mathfrak{H}_{2}} \subseteq K$. Но в таком случае $W / K \cong H \in \mathfrak{H}_{2}$. Получаем противоречие с выбором $H$. Следовательно, $\mathfrak{H}_{1} \subseteq \mathfrak{S}_{2}$ и, в силу симметричности, $\mathfrak{S}_{1}=\mathfrak{S}_{2}$. Лемма доказана.

Лемма 17. Пусть $\mathfrak{F}=\mathfrak{N}_{\pi} \mathfrak{H}-\Omega$-расслоенная формаџия с направлением $\beta,|\pi|>1$, $\pi(\Omega) \subseteq \pi$ и K( $\mathfrak{H}) \cap \mathfrak{U} \cap \Omega \subseteq \mathfrak{N}_{\pi}$. Тогда

$$
\mathfrak{S}=R_{0}\left(\bigcup_{A \in(K(\mathfrak{F}) \cap \Omega)} f(A)\right),
$$

где $f$ - минимальный внутренний $\Omega$-спутник формации $\mathfrak{F}$.

Доказательство. Введем обозначение

$$
\mathfrak{X}=\bigcup_{A \in(K(\mathfrak{F}) \cap \Omega)} f(A) .
$$

Предположим, что $\mathfrak{H} \nsubseteq R_{0}(\mathfrak{X})$ и пусть $G-$ группа минимального порядка из $\mathfrak{H} \backslash R_{0}(\mathfrak{X})$. Тогда $G-$ монолитическая группа с монолитом $R$. Поскольку $\mathfrak{S} \subseteq \mathfrak{F}$, справедливо включение $G \in \mathfrak{F}$. Очевидно, что $\mathfrak{N}_{\pi}$ имеет минимальный внутренний $\Omega$-спутник $m$ такой, что $m(A)=1$ для всех $A \in K\left(\Re_{\pi}\right) \cap \Omega$. В случае $K(R) \cap \Omega=\varnothing$ справедливо равенство $O_{B}(G)=1$ для любой группы $B \in \Omega$. По лемме 8 , 买 обладает $\Omega$-спутником $h$ таким, что $h(A)=\mathfrak{H}$ для всех $A \in K(\mathfrak{F}) \cap \Omega$. Тогда, по лемме $10, G \in f\left(Z_{p}\right)$ для некоторого $Z_{p} \in \mathfrak{N}_{\pi} \cap \Omega$, что противоречит выбору $G$. Значит, $K(R) \subseteq \Omega$.

Если $R$ - неабелева группа и $K(R)=(B)$, то $G / O_{B^{\prime}}(G) \cong G \in f(B) \subseteq R_{0}(\mathfrak{X )}$. Получаем противоречие. Значит $R$ - элементарная абелева $p$-группа для некоторого простого числа $p$. Рассмотрим регулярное сплетение

$$
T=Z_{q} \prec G=[K] G,
$$

где $Z_{q} \in \Omega, q \neq p$, а $K-$ база сплетения. Поскольку $O_{q}(G)=1$, по лемме 2 в [11],

$$
T_{\gamma\left(Z_{q}\right)}=O_{Z_{q}}(T)=K=T_{\alpha\left(Z_{q}\right)}=T_{\beta\left(Z_{q}\right)} .
$$

Так как $T / K \cong G \in \mathfrak{H}$, справедливо включение $T \subseteq \mathfrak{N}_{\pi} \mathfrak{H}=\mathfrak{F}$. Следовательно,

$$
T / T_{\beta\left(Z_{q}\right)}=T / K \cong G \in f\left(Z_{q}\right) \subseteq R_{0}(\mathfrak{X}) .
$$

Получаем противоречие. Поэтому $\mathfrak{S} \subseteq R_{0}(\mathfrak{X})$.

Обратное включение следует из леммы 8. Лемма доказана.

Лемма 18. Пусть $\mathfrak{F}=\mathfrak{N}_{p} \mathfrak{H}-$ несократимая факторизация однопорожденной $\Omega$-расслоенной формации $\mathfrak{F}$ с направлением $\beta$, причем $Z_{p} \in \Omega$. Тогда

$$
\mathfrak{H} / O_{p}(\mathfrak{H})=f\left(Z_{p}\right),
$$

где $f$-минимальный внутренний $\Omega$-спутник формаџиии $\mathfrak{F}$. 
Доказательство. По теореме $1, K(\mathfrak{S}) \cap \mathfrak{U} \cap \Omega \subseteq\left(Z_{p}\right)$. Тогда, по лемме 8, формация $\mathfrak{F}$ обладает $\Omega$-спутником $h$ таким, что $h\left(Z_{p}\right)=\mathfrak{H}$. Пусть $H$ произвольная группа из $\mathfrak{H}$, тогда $H / O_{p}(H) \in \mathfrak{S}=h\left(Z_{p}\right)$. Поскольку $O_{p}\left(H / O_{p}(H)\right)=1$, по лемме $10, H / O_{p}(H) \in f\left(Z_{p}\right)$. Следовательно, $\mathfrak{5} / O_{p}(\mathfrak{H}) \subseteq f\left(Z_{p}\right)$.

Несложно показать, что

$$
\mathfrak{F}=\mathfrak{N}_{p} \mathfrak{H}=\mathfrak{N}_{p}\left(\mathfrak{H} / O_{p}(\mathfrak{H})\right)
$$

Тогда, в силу леммы $8, f\left(Z_{p}\right) \subseteq \mathfrak{H} / O_{p}(\mathfrak{H})$. Значит, $\mathfrak{H} / O_{p}(\mathfrak{S})=f\left(Z_{p}\right)$. Лемма доказана.

Теорема 3. Пусть однопорожденная $\Omega$-расслоенная формащия $\mathfrak{F}$ с направлением $\beta$ допускает несократимую факторизаџию $\mathfrak{F}=\mathfrak{F}_{1} \mathfrak{F}_{2} \ldots \mathfrak{F}_{t}$, где $\mathfrak{F}_{1}, \mathfrak{F}_{2}, \ldots, \mathfrak{F}_{t}-$ неразложимье формации и формаџия $\mathfrak{F}_{1}$ также является $\Omega$-расслоенной с направлением $\beta$. Если формация $\mathfrak{F}_{1} \neq \mathfrak{N}_{p}$, где $Z_{p} \in \Omega$, то все множители факторизации $\mathfrak{F}$ однозначно определень.

Доказательство. Допустим, что формация $\mathfrak{F}$ имеет две различные несократимые факторизации $\mathfrak{F}=\mathfrak{M}_{1} \mathfrak{M}_{2} \ldots \mathfrak{M}_{t}=\mathfrak{S}_{1} \mathfrak{S}_{2} \ldots \mathfrak{H}_{r}$ с неразложимыми множителями, в которых $\mathfrak{M}_{1}$ и $\mathfrak{H}_{1}-\Omega$-расслоенные формации с направлением $\beta$. Из теоремы 2 следует, что $t \leqslant 3$ и $r \leqslant 3$.

Рассмотрим случай, когда $t=r=2$, то есть $\mathfrak{F}=\mathfrak{M}_{1} \mathfrak{M}_{2}=\mathfrak{H}_{1} \mathfrak{H}_{2}$. Если $\mathfrak{M}_{1}$ и $\mathfrak{H}_{1}$ не нильпотентны, то, по лемме $16, \mathfrak{M}_{2}=\mathfrak{S}_{2}$. Из теоремы 2 следует, что $\mathfrak{M}_{2}$ и $\mathfrak{H}_{2}-$ однопорожденные абелевы формации. Тогда, по лемме 3.4 .5 в [12], $\mathfrak{M}_{1}=\mathfrak{S}_{1}$. Получили противоречие, следовательно, по крайней мере одна из формаций $\mathfrak{M}_{1}$ и $\mathfrak{H}_{1}$ нильпотентна.

Допустим, что нильпотентны обе формации. По теореме $2, \mathfrak{M}_{1} \subseteq \mathfrak{N}_{\omega} \mathfrak{N}_{\omega^{\prime}}$, где $\omega=\pi(\Omega \cap \mathfrak{U})$ и $\mathfrak{H}_{1} \subseteq \mathfrak{N}_{\omega} \mathfrak{N}_{\omega^{\prime}}$. Если $\mathfrak{M}_{1} \subseteq \mathfrak{N}_{\omega}$ и $\mathfrak{H}_{1} \subseteq \mathfrak{N}_{\omega}$, то $\mathfrak{M}_{1}=\mathfrak{H}_{1}=\mathfrak{N}_{\pi}$, где $\pi=\pi\left(\mathfrak{M}_{1}\right)$. Тогда, в силу леммы $17, \mathfrak{M}_{2}=\mathfrak{H}_{2}$. Получаем противоречие. Предположим, что $\mathfrak{M}_{1} \subseteq \mathfrak{N}_{\omega}$, а $\mathfrak{H}_{1} \nsubseteq \mathfrak{N}_{\omega}$, тогда $\mathfrak{M}_{1} \subseteq \mathfrak{S}_{1}$. Пусть $G$ - группа минимального порядка из разности $\mathfrak{S}_{1} \backslash \mathfrak{M}_{1}$, в таком случае $G-$ монолитическая $q$-группа, $Z_{q} \notin \Omega$. По лемме 18.8 из [9], для некоторого $p \in \omega \cap \pi\left(\mathfrak{M}_{1}\right)$ существует точный неприводимый $F_{p}[G]$-модуль $T$. Рассмотрим полупрямое произведение $M=[T] G$. Поскольку $M / T \cong G \in \mathfrak{M}_{2}$, справедливы соотношения $M^{\mathfrak{M}_{2}} \subseteq T \in \mathfrak{M}_{1}$ и $M \in \mathfrak{M}_{1} \mathfrak{M}_{2}=\mathfrak{H}_{1} \mathfrak{H}_{2}$. Из теоремы 2 следует, что $G \notin \mathfrak{H}_{2}$, значит, $M^{\mathfrak{H}_{2}} \nsubseteq T$ и $M^{\mathfrak{H}_{2}} \in \mathfrak{H}_{1} \subseteq \mathfrak{N}$. Получаем противоречие. Аналогично доказывается, что случай $\mathfrak{M}_{1} \nsubseteq \mathfrak{N}_{\omega}$ и $\mathfrak{H}_{1} \nsubseteq \mathfrak{N}_{\omega}$ также невозможен. Таким образом, $\mathfrak{M}_{1}=\mathfrak{H}_{1}$.

Пусть $H-$ группа минимального порядка из разности $\mathfrak{S}_{2} \backslash \mathfrak{M}_{2}$. По теореме $2, \mathfrak{S}_{2}$ - абелева формация, значит, $H \cong Z_{q}$. Если $Z_{q} \notin \Omega$, то, по теореме $2, Z_{q} \in \mathfrak{M}_{1}$ и $Z_{q} \notin \mathfrak{H}_{1}$, то есть $\mathfrak{M}_{1} \neq \mathfrak{H}_{1}$. Получаем противоречие. Значит, $Z_{q} \in \Omega$. Пусть $M=[T] Z_{q}$, где $T$ - точный неприводимый $F_{p}\left[Z_{q}\right]$-модуль для некоторого $p \in \omega \cap \pi\left(\mathfrak{H}_{1}\right)$. Ясно, что $M \in \mathfrak{H}_{1} \mathfrak{H}_{2}=\mathfrak{M}_{1} \mathfrak{M}_{2}$. Но $Z_{q} \notin \mathfrak{M}_{2}$, поэтому $M^{\mathfrak{M}_{2}} \nsubseteq T$ и $M^{\mathfrak{M}_{2}} \in \mathfrak{M}_{1} \subseteq \mathfrak{N}$. Получаем противоречие. Следовательно, $\mathfrak{H}_{2} \subseteq \mathfrak{M}_{2}$. В силу симметрии, $\mathfrak{H}_{2}=\mathfrak{M}_{2}$. Получаем противоречие.

Остается предположить, что $\mathfrak{H}_{1} \subseteq \mathfrak{N}$, а $\mathfrak{M}_{1} \nsubseteq \mathfrak{N}$. Обозначим $m_{1}$ и $f$ минимальные $\Omega$-спутники формаций $\mathfrak{M}_{1}$ и $\mathfrak{F}$ соответственно. Поскольку $\mathfrak{M}_{1} \nsubseteq \mathfrak{N}$, найдется такая группа $Z_{q} \in \Omega$, что $m_{1}\left(Z_{q}\right) \neq \mathfrak{M}_{0}$. Из теоремы 5 в [4] и леммы 10 вытекает, что $m_{1}\left(Z_{q}\right)$ порождается некоторым множеством нильпотентных групп $G$ с $O_{Z_{q}}(G)=1$, то есть $m_{1}\left(Z_{q}\right) \subseteq \mathfrak{N}_{q^{\prime}}$. В силу леммы $8, \mathfrak{F}$ обладает таким $\Omega$-спутником $t$, что $t\left(Z_{p}\right)=m_{1}\left(Z_{p}\right) \mathfrak{M}_{2}$, если $Z_{p} \in K\left(\mathfrak{M}_{1}\right) \cap \Omega=K(\mathfrak{F}) \cap \Omega$. Пусть $N$ - монолитическая группа из $m_{1}\left(Z_{p}\right) \mathfrak{M}_{2}$. Если $O_{Z_{p}}(N)=1$, то, в силу леммы $10, N \in f\left(Z_{p}\right)$. Пусть $O_{Z_{p}}(N) \neq 1$. По определению, 
$N^{\mathfrak{M}_{2}} \in m_{1}\left(Z_{p}\right) \subseteq \mathfrak{M}_{p^{\prime}}$. Значит, $N^{\mathfrak{M}_{2}}=1$ и $N \in \mathfrak{M}_{2}$. Для произвольной группы $B$ из $m_{1}\left(Z_{p}\right)$ рассмотрим регулярное сплетение

$$
W=B \imath N=[K] N,
$$

где $K-$ база сплетения. Ясно, что $W \in m_{1}\left(Z_{p}\right) \mathfrak{M}_{2}=t\left(Z_{p}\right)$. В силу леммы $10, W \in f\left(Z_{p}\right)$ и $N \in f\left(Z_{p}\right)$. Таким образом, $m_{1}\left(Z_{p}\right) \mathfrak{M}_{2} \subseteq f\left(Z_{p}\right)$, а так как $f \leqslant t$, справедливо равенство

$$
m_{1}\left(Z_{p}\right) \mathfrak{M}_{2}=f\left(Z_{p}\right)
$$

По лемме 17,

$$
\begin{aligned}
\mathfrak{H}_{2} & =R_{0}\left(\bigcup_{A \in(K(\mathfrak{F}) \cap \Omega)} f(A)\right)=Q R_{0}\left(\bigcup_{A \in(K(\mathfrak{F}) \cap \Omega)} m_{1}(A) \mathfrak{M}_{2}\right) \\
& =\bigvee_{A \in(K(\mathfrak{F}) \cap \Omega)} m_{1}(A) \mathfrak{M}_{2} .
\end{aligned}
$$

По лемме 3.4 .4 в [12],

$$
\mathfrak{H}_{2}=\left(\bigvee_{A \in(K(\mathfrak{F}) \cap \Omega)} m_{1}(A)\right) \mathfrak{M}_{2}, \quad \bigvee_{A \in(K(\mathfrak{F}) \cap \Omega)} m_{1}(A) \neq 1 .
$$

Получили противоречие с неразложимостью $\mathfrak{S}_{2}$.

Допустим, что $t=r=3$, то есть $\mathfrak{F}=\mathfrak{M}_{1} \mathfrak{M}_{2} \mathfrak{M}_{3}=\mathfrak{H}_{1} \mathfrak{H}_{2} \mathfrak{H}_{3}$. По лемме 3.2 .5 в [12], формации $\mathfrak{M}_{1} \mathfrak{M}_{2}$ и $\mathfrak{S}_{1} \mathfrak{H}_{2}$ не нильпотентны. Поэтому, по лемме $16, \mathfrak{M}_{3}=\mathfrak{H}_{3}$. По теореме $2, \mathfrak{M}_{1} \subseteq \mathfrak{N}_{\omega}$ и $\mathfrak{H}_{1} \subseteq \mathfrak{N}_{\omega}$, значит, $\mathfrak{M}_{1}=\mathfrak{H}_{1}=\mathfrak{N}_{\pi}$. Допустим, что существует группа $G \in \mathfrak{M}_{2} \backslash \mathfrak{H}_{2}$. По лемме $17, \mathfrak{M}_{1} \mathfrak{M}_{2}=\mathfrak{S}_{1} \mathfrak{H}_{2}$, поэтому $G^{\mathfrak{S}_{2}} \in \mathfrak{S}_{1} \subseteq \mathfrak{N}_{\omega}$. Но из

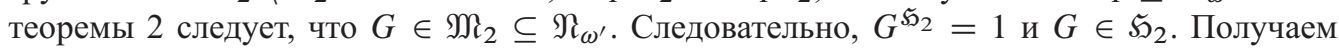
противоречие. Таким образом, $\mathfrak{M}_{2} \subseteq \mathfrak{S}_{2}$ и, из соображений симметрии, $\mathfrak{M}_{2}=\mathfrak{S}_{2}$.

Остается случай $t \neq r$, например, $\mathfrak{F}=\mathfrak{M}_{1} \mathfrak{M}_{2} \mathfrak{M}_{3}=\mathfrak{H}_{1} \mathfrak{H}_{2}$. Если $\mathfrak{H}_{1} \nsubseteq \mathfrak{N}$, то, по лемме $16, \mathfrak{M}_{3}=\mathfrak{S}_{2}$. Из теоремы 2 следует, что $\mathfrak{M}_{3}=\mathfrak{S}_{2}-$ однопорожденная абелева формация. Значит, по лемме $17, \mathfrak{M}_{1} \mathfrak{M}_{2}=\mathfrak{H}_{1}$, что противоречит неразложимости формации $\mathfrak{H}_{1}$. Следовательно, $\mathfrak{H}_{1} \subseteq \mathfrak{N}$. Поскольку $\mathfrak{M}_{1} \subseteq \mathfrak{N}_{\omega}$, справедливы соотношения $\mathfrak{H}_{1} \nsubseteq \mathfrak{N}_{\omega^{\prime}}$ и $\mathfrak{M}_{1} \subseteq \mathfrak{H}_{1}$. Если $H-$ группа из разности $\mathfrak{H}_{1} \backslash \mathfrak{M}_{1}$, то, по теореме 2 , $H \cong Z_{q} \notin \Omega$. В этом случае существует точный неприводимый $F_{p}\left[Z_{q}\right]$-модуль $T$ для некоторого $p \in \omega \cap \pi\left(\mathfrak{H}_{1}\right)$. Пусть $M=[T] Z_{q}$. Очевидно, что $M \in \mathfrak{M}_{1} \mathfrak{M}_{2} \mathfrak{M}_{3}=\mathfrak{H}_{1} \mathfrak{H}_{2}$. Но $Z_{q} \notin \mathfrak{H}_{2}$, поэтому $M^{\mathfrak{H}_{2}}=M \in \mathfrak{H}_{1}$, что противоречит нильпотентности $\mathfrak{H}_{1}$. Таким образом, $\mathfrak{M}_{1}=\mathfrak{H}_{1} \subseteq \mathfrak{N}_{\omega}$.

Предположим, что найдется группа $S \in \mathfrak{M}_{3} \backslash \mathfrak{H}_{2}$. Нетрудно показать, что $S \cong Z_{q} \in \Omega$. Из теоремы 2 вытекает, что $\mathfrak{M}_{2} \subseteq \mathfrak{N}_{\omega^{\prime}}$ и $\mathfrak{M}_{2} \subseteq \mathfrak{H}_{2}$. Для произвольного числа $p \in \pi\left(\mathfrak{M}_{2}\right)$ существует точный неприводимый $F_{p}\left(Z_{q}\right)$-модуль $T$. Пусть $M=[T] Z_{q}$, тогда $M \in \mathfrak{M}_{2} \mathfrak{M}_{3} \subseteq \mathfrak{H}_{1} \mathfrak{H}_{2}$. Но $Z_{q} \notin \mathfrak{H}_{2}$, значит, $M \in \mathfrak{H}_{1}$. Получаем противоречие с нильпотентностью $\mathfrak{H}_{1}$, то есть $\mathfrak{M}_{3} \subseteq \mathfrak{H}_{2}$.

Возьмем произвольную группу $D$ из разности $\mathfrak{M}_{2} \mathfrak{M}_{3} \backslash \mathfrak{H}_{2}$. Ясно, что $D \in \mathfrak{H}_{1} \mathfrak{H}_{2}$ и монолит $D^{\mathfrak{H}_{2}} \in \mathfrak{H}_{1} \subseteq \mathfrak{N}_{\omega}$. Но $D^{\mathfrak{M}_{3}} \neq 1$ и $D^{\mathfrak{H}_{2}} \subseteq D^{\mathfrak{M}_{3}} \in \mathfrak{M}_{2} \subseteq \mathfrak{N}_{\omega^{\prime}}$. Из данного противоречия заключаем, что $\mathfrak{M}_{2} \mathfrak{M}_{3} \subseteq \mathfrak{S}_{2}$. Пусть $C$ - группа минимального порядка из разности $\mathfrak{H}_{2} \backslash \mathfrak{M}_{2} \mathfrak{M}_{3}$. Тогда $C-$ монолитическая группа с монолитом $R$. Поскольку 


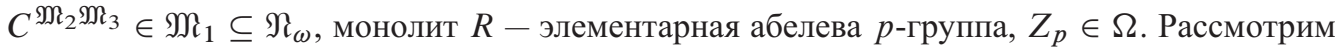
$M=[T] C$, где $T$ - точный неприводимый $F_{q}[C]$-модуль для некоторого $q \in \pi\left(\mathfrak{S}_{1}\right) \backslash\{p\}$. Очевидно, что $M \in \mathfrak{H}_{1} \mathfrak{H}_{2}=\mathfrak{M}_{1} \mathfrak{M}_{2} \mathfrak{M}_{3}$ и $M^{\mathfrak{M}_{2} \mathfrak{M}_{3}} \in \mathfrak{M}_{1} \subseteq \mathfrak{N}_{\omega}$. С другой стороны, $M^{\mathfrak{M}_{2} \mathfrak{M}_{3}} \nsubseteq T$, что противоречит нильпотентности $M^{\mathfrak{M}_{2} \mathfrak{M}_{3}}$. Итак, $\mathfrak{M}_{2} \mathfrak{M}_{3}=\mathfrak{S}_{2}$. Теорема доказана.

С использованием леммы 18 аналогично доказывается следующая теорема.

Теорема 4. Пусть однопорожденная $\Omega$-расслоенная формаџия $\mathfrak{F}$ с направлением $\beta$ допускает несократимую факторизацию $\mathfrak{F}=\mathfrak{N}_{p} \mathfrak{F}_{2} \ldots \mathfrak{F}_{t}=\mathfrak{N}_{p}\left(\mathfrak{F}_{2} / O_{p}\left(\mathfrak{F}_{2}\right)\right) \mathfrak{F}_{3} \ldots \mathfrak{F}_{t}$, где $\mathfrak{F}_{2} / O_{p}\left(\mathfrak{F}_{2}\right), \mathfrak{F}_{3}, \ldots, \mathfrak{F}_{t}-$ неразложимые формации, $Z_{p} \in \Omega$. Тогда множители $\mathfrak{F}_{2} / O_{p}\left(\mathfrak{F}_{2}\right), \mathfrak{F}_{3}, \ldots, \mathfrak{F}_{t}$ факторизащии $\mathfrak{F}$ однозначно определеньл.

\section{Список литературы}

1. Скиба А. Н., О произведении формаций. Алгебра и логика (1983) 22, №5, 574-583.

2. Шеметков Л. А., Экраны произведения формаций. Доклады АН Белорусской ССР (1981) 25, №8, 677-680.

3. Skiba A. N., On nontrivial factorizations of a one-generated local formation of finite groups. Contemp. Math. (1992) 131, №1, 363-374.

4. Ведерников В. А., Сорокина М. М., $\Omega$-расслоенные формации и классы Фиттинга конечных групп. Дискретная математика (2001) 13, №3, 125-144.

5. Vedernikov V. A., Maximal satellites of $\Omega$-foliated formations and Fitting classes. Proc. Steklov Inst. Math. (2001) 217-233.

6. Скиба А. Н., Шеметков Л. А., Кратно $\omega$-локальные формации и классы Фиттинга конечных групп. Матем. Труды (1999) 2, №2, 114-147.

7. Ведерников В. А., Коптюх Д. Г., Частично композищионные формации групn, Препринт №2. БГПУ, Брянск, 1999.

8. Еловиков А. Б., Факторизация однопорожденных формаций. Математические заметки (2003) 73, №5, 684-697.

9. Шеметков Л. А., Скиба А. Н., Формаџии алгебраических систем. Наука, Москва, 1989.

10. Шеметков Л. А., Формации конечных групn. Наука, Москва, 1978.

11. Скиба А. Н., Шеметков Л. А., О минимальном композиционном экране композиционной формации. Вопросы алгебры (1992) 7, 39-43.

12. Скиба А. Н., Алгебра формаций. Беларуская навука, Минск, 1997.

13. Doerk K., Hawkes T., Finite soluble groups. Walter de Gruyter, Berlin, 1992.

14. Сорокина М. М., Корпачева М. А., О критических $\Omega$-расслоенных формациях конечных групп. Дискретная математика (2006) 18, №1, 106-115.

Статья поступила 20.12.2006.

Переработанный вариант поступил 10.02.2009. 